\title{
Die Entstehung des Knochengewebes und des Zahnbeins.
}

Ein Beitrag zur Lehre von der Bildung der Grundsubstanzen.

Von

J. Disse, Marburg.

Hierzn Tafel XXI und XXII.

Die Frage nach der Bildung der Interzellularsubstanz ist fïr die Zellentheorie gerade so wichtig wie die nach der Bildung der Zellen selbst. Bei der Aufstellung der Zellentheorie wurde von Schwann und von seinen Mitarbeitern die Interzellularsubstanz als das Wichtigste angesehen; „die Interzellularsubstanz ist das Ursprüngliche, identisch mit dem Cytoblastem; Cytoblastem ist die Materie, in und aus welcher die Zellen sich entwickeln, und was nach Vollendung und Umbildung der Zellen von der ursprünglichen Materie übrig bleibt, ist Interzellularsubstanz" (Henle, Allgemeine Anatomie, S. 214).

Diese Lehre war von kurzer Dauer; die Beobachtungen, aus denen man die freie Zellbildung gefolgert hatte, mussten eine andere Deutung erfahren, und es wurde die Existenz eines "Cytoblastems“ selbst fraglich, als an die Stelle der Lehre von der freien Zellbildung der Satz trat "Omnis cellula a cellula“. Wenn eine jede Zelle von einer bereits vorhandenen gebildet wird, ist eine ungeformte, aber zur Zellenbildung befähigte Substanz überflüssig. Es gibt dann nur ein einziges Bildungselement für die Gewebe des Organismus, die Zelle selbst. Die Zelle ist das ursprünglich Vorhandene; und wenn wir ausser ihr eine "Zwischensubstanz" vorfinden, so kann diese nicht den "Rest der ursprünglich vorhandenen Materie" darstellen, der nach der Bildung der Zellen übrig geblieben ist, sie muss vielmebr eine andere Herkunft haben. In irgend einer Weise muss die Zwischensubstanz auf die Zellen zurückgeführt werden können.

An Versuchen, die Entstehung der Interzellularsubstanz aufzuklären, hat es nicht gefehlt; es entsprach den Verhältnissen, dass derartige Untersuchungen vorwiegend an denjenigen Geweben angestellt wurden, die sich durch das Vorwiegen der Interzellular- 
substanz, durch das Zurücktreten der Zellen selbst auszeichnen. Wie bildet sich die Interzellularsubstanz des Bindegewebes, des Knorpels, des Knochens? So wurde gefragt; und da die Interzellularsubstanz nicht amorph, sondern geformt ist, da sie im Bindegewebe, Knochen und Zahnbein leimgebende Fibrillen in bestimmter Anordnung enthält, so wurde besonders für das Bindegewebe die Frage aufgeworfen: Wie entstehen die Fibrillen?

Entstehen sie durch eine Differenzierung, eine Art von „Prägung" in einer von den Zellen der ersten Anlage ausgeschiedenen Substanz, also ausserhalb der Zellen, oder entstehen sie im Protoplasma der Zellen selbst?

In jedem Falle blieb es verständlich, dass bei der Bildung der Fibrillen die Zellen des Gewebes erbalten bleiben. Es wurde aber bei den Untersuchungen über die Bildung der Fibrillen die ungeformte Substanz, die ausser den Fibrillen in wechselnder Menge in den Unterabteilungen des Bindegewebes vorhanden ist, nicht berücksichtigt; wohl deshalb, weil sie amorph ist und viel weniger ins Auge fällt als die strukturierten Elemente der Interzellularsubstanz.

Bekanntlich hat erst v. Ebner festgestellt (Über den feineren Bau der Knochensubstanz. Wiener Sitzungsberichte, Bd. 72, 1876), dass auch das Knochengewebe und das Dentin leimgebende Fibrillen enthalten, die in eine verkalkte Grundsubstanz eingelagert sind. - Vor seinen Untersuchungen galten Knochen und Zahnbein als einfache, nicht weiter strukturierte Grundsubstanzen. Bei der Untersuchung der Entstehung derartiger Gewebe konnte es sich nicht um Fibrillenbildung handeln; es lag vielmehr der einfache Fall vor von Produktion einer ungeformten Grundsubstanz.

Nun hatte Heinrich Müller gezeigt (Über die Entwicklung der Knochensubstanz. Zeitschr. f. wiss. Zoologie, Bd. 9, 1858), dass bei den knorplig vorgebildeten Skeletteilen das Knochengewebe nicht etwa durch Umwandlung des Knorpels sich bildet, sondern dass es eine Neubildung darstellt, die an die Stelle des einschmelzenden Knorpels tritt. Ge genbaur (Über die Bildung des Knochengewebes. I. Jenaische Zeitschrift, Bd. 1, 1864. II. Ebenda, Bd. 3, 1867), konnte den Nachweis führen, dass das Auftreten des Knochengewebes geknüpft ist an das Auftreten bestimmter Zellen, der Osteoblasten, und dass aller Wahrscheinlichkeit 
nach lediglich diese Zellen das Material liefern, aus dem sich das Knochengewebe bildet. Die Zellen selbst bleiben bei diesem Prozess erbalten und werden zu den Knochenzellen; sie sezernieren einen Stoff, der zur Grundsubstanz des Knochens wird. Morphologisch bestände also niemals ein Zusammenhang zwischen den Osteoblasten und dem Knochengewebe; dieses ginge aus einem Sekret der Osteoblasten hervor.

Fast gleichzeitig batte Waldeyer die Bildung des Knochengewebes untersucht (Über den Ossifikationsprozess. Arch. f. mikr. Anat., Bd. 1, 1865); er konnte bestätigen, dass das Knochengewebe von den Osteoblasten geliefert wird. Über die Art der Bildung des Gewebes aber war er anderer Ansicht als Gegenbaur. Waldeyer fand eine direkte Beziehung des Knochengewebes zu den Osteoblasten; diese Zellen gehen mit ihrem Protoplasma unmittelbar in das Knochengewebe über. Das Knochengewebe entsteht nicht aus einem von den Osteoblasten abgesonderten Sekret, sondern es ist umgewandeltes Zellprotoplasma. Die Zellen innerhalb der Knochenhöhlen stammen zwar von den Osteoblasten $a b$; indessen stellt eine Knochenzelle nicht einen ganzen Osteoblasten vor, sondern einen "reduzierten", dessen Protoplasma zum grössten Teil zu Knochengewebe geworden ist.

Waldeyer entdeckte ferner, dass das Zahnbein ebenso auf bestimmte Zellen zurückzuführen ist, wie das Knochengewebe auf die Osteoblasten (Untersuchungen über die Entwicklung der Zahne. I. Königsberger med. Jahrbücher, 4. Bd., 1864. II. Zeitschr. f. rationelle Medizin. III. R., Bd.24, 1865). Wo sich Zabnbein bilden soll, treten in zusammenhängender Lage wohl charakterisierte Zellen auf, die „Dentinzellen“ oder „Odontoblasten“. Das Protoplasma dieser Zellen wird, bis auf einen Rest, zur Grundsubstanz des Dentins. Der Rest des Protoplasmas nebst dem Kern erhält sich an der inneren Fläche der Dentinlage und sendet in diese die Zahnfaser hinein.

Gegenbaur schildert das tatsächliche Verhalten der jungen Knochensubstanz zu den Osteoblasten folgendermassen: „Die erste Entwicklung der Knochenbälkchen des Scheitel- oder Stirnbeins erfolgt innerhalb einer ganz kontinuierlichen Schicht von Zellen . . . zwischen diesen Zellen sondert sich eine alsbald sklerosierende, eckig geformte Masse $a b$, die von den Zellen allseitig umgeben 
wird, und durch fernere Abscheidung von seiten der Zellen weiter wächst." Diese Anlage besitzt keine Struktur, sie nimmt an Masse beständig zu und schliesst einzelne Osteoblasten ein. Der Modus der Absonderung ist nicht beobachtet worden.

Demgegenüber betont Waldeyer, dass das Knochengewebe gerade so entsteht wie das faserige Bindegewebe. Dieses bildet sich „aus einer formalen und chemischen Umwandlung eines Teils des Protoplasmas der embryonalen Bildungszellen, indem dasselle leimgebend und zugleich mehr oder weniger tibrillär wird* Viele Bildungszellen gehen dabei ganz zu Grunde; andere wandeln nur die Aussenschichte ihres Protoplasmas in Bindegewebe um, wäbrend das den Kern umgebende Protoplasma sich erlaalt und zu einer Bindegewebszelle wird. Nun sind die Osteoblasten "die embryonalen Bildungszellen des Ḱnochengewebes: ein 'Teil derselben geht „mit Schwund des Kerns ganz die Umwandlung in leimgebendes faseriges Gewebe ein . . . von einem anderen Teil tun das nur die peripheren I'rotoplasmaschichten. Der Rest bleibt als kernhaltiges Knochenkörperchen zurück-. (S. 359.)

Die erst gebildete Knochensubstanz tritt nach Waldeyers Angabe "als ein leicht gelblicher, homogen aussebender Streifen" as den Rändern der Knorpelbalken auf. In diese Knocbensubstanz geht hier und da das Protoplasma eines Osteoblasten direkt über. „Bei einzelnen Osteoblasten veründern sich die peripherischen T'eile, indem sie ihr dunkel gekörntes Ausseben verlieren ... Nur die dicht um den Kern gelegenen Teile des Protoplasmas bleiben unverändert."

Die verănderten peripheren Partien der Osteoblasten sieht man „unmittelbar in bereits fertige Knochensubstanz übergehen " (S. 363). Das sind positive, auch durch Abbildungen belegte Angaben, die für einen direkten Übergang des Protoplasmas der Osteoblasten in Knochengewebe sprechen.

$\mathrm{Zu}$ dem gleichen Resultat ist Stieda gekommen. (Die Entwicklung des Knochengewebes. 1872.) Stieda vertritt die Lehre, dass "das ächte Knochengewebe sich stets obne Beteiligung des Knorpels bilde", dass es also überall da, wo es auftritt, eine Neubildung repräsentiert. Sowohl an Stelle atrophierenden Knorpels als innerhalb bindegewebiger Membranen entsteht das Knochengewebe durch die "Metamorphose" der Osteoblasten. 
Ein Abschnitt eines Osteoblasten wandelt sich in Knochengewebe nm; ein anderer bleibt unverändert. "Während der aussere Abschnitt einer jeden einzelnen Zelle durch einen noch nicht erklärten Prozess fest wird - sklerosiert - bleibt der Kern mit dem Rest des unveränderten Protoplasma weich. Die Summe des sklerosierten Protoplasma einer Anzahl von Osteoblasten gibt die sogenannte Grund- oder Zwischensubstanz des Knochengewebes; der Kern nebst anhängendem Protoplasma ist das sogenannte Kinochenkörperchen." Die sklerosierten Abschnitte der Osteoblasten sind daran kenntlich, dass sie bei Karminfärbung hell bleiben, wahrend der Kern und das unverannderte Protoplasma sich färben.

'Trotz dieser Bestätigung durch stieda wurde die Lehre von Waldeyer nicht allseitig anerkannt; die meisten Forscher schlossen sich an Gegenbaur an und betrachteten das Knochengewebe als ein Sekret der Osteoblasten. Sie stützten sich darauf, dass eine direkte Beteiligung des Protoplasmas dieser Zellen an der Bildung des Knochengewebes nicht zu erkennen sei; die Osteoblasten waren stets vom jungen Knochengewebe getrennt, und man könne keine Verkleinerung derselben während der Knochenbildung nachweisen. Es müsse aber eine Verkleinerung der Osteoblasten eintreten, wenn wirklich ein Teil des Protoplasmas einer jeden Zelle zur Bildung der Grundsubstanz verwendet würde.

In der späteren Zeit, nach dem Erscheinen der Arbeiten von Gegenbaur, Waldeyer und Stieda, ist keine Beobachtung bekannt geworden, die eine Entscheidung zu Gunsten der Lehre von Gegenbaur oder der Theorie von Waldeyer zu treffen erlaubt hätte. Es scheint, dass, mit Ausnahme von Stieda, keiner der späteren Untersucher Bilder gesehen hat, die die Osteoblasten im Zusammenhang mit jungem Knochengewebe zeigten.

Und doch sind die Angaben von Waldeyer über die Veränderungen der Osteoblasten von der grössten Wichtigkeit. Die Osteoblasten trennen sich in eine körnige Innenzone und eine mehr homogene Aussenzone; nur diese geht in die Grundsubstanz des Knochens über. Es war ein positiver Beweis für die Richtigkeit der Lehre von Max Schultze, dass „der grösste Teil der sogenannten Interzellularsubstanzen aus umgewandelter Zellsubstanz besteht" (Über Muskelkörperchen. Reicherts Archiv 1861). 
Diese Angaben hätten nachgeprüft werden müssen, statt mit Stillschweigen übergangen zu werden. Man wandte sich lieber der Frage nach der Entstehung der Bindegewebsbündel zu. Diese wenigstens sollten, so suchte man $\mathrm{zu}$ erweisen, direkt aus dem Protoplasma der Bildungszellen hervorgehen. Franz Boll formulierte im Jabre 1872 (Untersuchungen über die Entwicklung der Gewebe. Arch. f. mikr. Anat., Bd. 8) die von ihm im Anschluss an Max Schultze vertretenen Ansichten folgendermassen: „Das Protoplasma der Embryonalzellen bildet die Fibrillen auf seiner Oberflăche und aus seiner Substanz . . . indessen ist die Fibrillensubstanz etwas Neues, durch die formative Tatigkeit des Protoplasma Gebildetes". Auf die Frage nach der Entstehung der Grundsubstanz des Bindegewebes ist weder F. Boll noch die späteren Bearbeiter der gleichen Aufgabe eingegangen. Immer lautete die Fragestellung nur: Entstehen die Fasern des Bindegewebes innerhalb der Zellen, im Protoplasma, oder entstehen sie innerhalb einer zwischen den Zellen gelegenen, amorphen Grundsubstanz? Wie diese Grundsubstanz selbst entsteht, wurde nicht weiter untersucht.

Definieren wir mit Waldeyer (Kittsubstanz und Grundsubstanz, Epithel und Endothel. Arch. f. mikr. Anat., Bd.57, 1901) die Grundsubstanz als ,eine homogene und strukturlose Bildung, in welche Zellen und Fasern des betreffenden Gewebes eingelagert sind", so müssen wir feststellen, dass im Knochengewebe und im Zahnbein der Grundsubstanz eine viel grössere Bedeutung zukommt, als im fibrillären Bindegewebe. Die leimgebenden Fasern, die in die Grundsubstanz eingelagert sind, treten dieser gegenüber zurück. Besonders muss betont werden, dass bei der Bildung von Knochen und von Zahnbein anfanglich nur die Grundsubstanz vorhanden ist; Fasern treten erst auf; wenn die Grundsubstanz als selbstăndige Lage erscheint. Will man also die Frage beantworten, wie Knochen und Zahnbein entstehen, so muss man untersuchen, wie die Grundsubstanz sich bildet. Dafür gilt die von Max Schultze prazisierte Fragestellung: Entsteht die Grundsubstanz durch Umwandlung des Protoplasmas bestimmter Zellen, oder entsteht sie als ein Sekret, als eine tlüssige, nach der Abscheidung erhärtende Masse?

Für die Bildung der Grundsubstanz kommen beim Knochengewebe nur die Osteoblasten, beim Zahnbein die Dentin- 
zellen in Betracht. Es handelt sich nicht darum, die Herkunft dieser Zellformen zu untersuchen, sondern festzustellen, ob die Bildung der Grundsubstanz des Knochens durch die Osteoblasten, des Zahnbeins durch die Dentinzellen erwiesen werden kann. Und wenn das der Fall ist: wird die Grundsubstanz durch ein erhärtendes Sekret geliefert, oder geht sie aus umgewandeltem Protoplasma hervor?

Die Fragen sind ja schon von Gegenbaur und von Waldeyer aufgeworfen und derart beantwortet worden, dass Waldeyer die Grundsubstanz für umgewandeltes Protoplasma; Gegenbaur sie für ein Sekret erklart hat. Die späteren Untersucher haben sich teils für Wald ey er, teils für G eg e n ba u r erklärt. Kölliker (Gewebelehre, 6. Aufl., Bd. I, S. 327) schloss sich der Lehre von Gegenbaur an; Spuler (Verhandl. der Anat. Gesellsch. 1899) steht mehr auf der Seite von Waldeyer. Nach seiner Angabe steht das jüngste Knochengewebe „in direktem Zusammenhang mit den in Fortsätze auslaufenden Osteoblasten". Er bezeichnet das jüngste Knochengewebe als „kollagene Substanz " und findet diese bald fibrillär, bald strukturlos.

Flemming (Histogenese der Kittsubstanzen. Hertwig, Handbuch der Entwicklungslebre, Bd. 3, II. Abt. 1902) ist nicht der Ansicht, dass die Beobachtungen erlaubten, die Frage nach der Entstehungsweise des Knochengewebes zu entscheiden; er lässt beide Möglichkeiten zu. Die Osteoblasten scheiden ,an ihrer Peripherie eine aus Knochenfibrillen und verkalkter Kittsubstanz bestehende Schale aus", oder formen diese aus ihrer Substanz. „Dass dies das Wesen des Vorgangs sei, können wir nur schliessen, ihn direkt $\mathrm{zu}$ beobachten, ist bis jetzt nicht wohl möglich."

Diese Auffassung ist zu resigniert; es ist, wie in folgendem gezeigt werden soll, recht wohl möglich, die Bildung der Grundsubstanz des Knochens aus Osteoblasten direkt zu sehen. Dazu ist gut konserviertes, in feine Schnitte zerlegbares Material erforderlich; beides erlaubt die moderne T'echnik herzustellen.

\section{Die Entstehung der Grundsubstanz des Knochen- gewebes.}

(Vgl. Fig. 1-13, Taf. XXI.)

Ich habe die Bildung des Knochengewebes bei der enchondralen Verknöcherung sowie bei der Knochenbildung in Membranen 
untersucht. Am geeignetsten erwiesen sich mir menschliche Embryonen, die auch W ald e y e r zum Studium der Verknöcherung benutzt hat. Besonders ein in Formol-Alkohol $(95 \%$ Alkohol 90 voll., Formol 10 voll.) fixierter menschlicher Embryo aus dem vierten Monat lieferte sehr klare und eindeutige Präparate über die Bildung der Grundsubstanz des Knochens. Für das Studium der Knochenbildung in Membranen wurden auch noch Schweineembryonen, in Sublimat-Eisessig fixiert, herangezogen, um $z u$ untersuchen, $o b$ auch bei diesen die am Menschen gefundenen Bildungsstadien zu sehen wären.

Als bestes Verfahren ergab sich folgendes: Der mit dem l'eriost herausgelöste Knochen - die Tibia - wurde in $10 \%$ Kochsalzlösung, der $2 \%$ Salzsäure zugesetzt waren, entkalkt. im ganzen mit Hämalaun durchgefürbt, gut in destilliertem Wasser extrahiert und in Paraffin eingeschmolzen. Schnitte von 5 "Dicke wurden auf dem Objektträger, nach Entfernung des Parafins, mit folgender Lösung nachgefärbt: Alkohol $95 \%$, 90 voll., Rubin S 1,0, Orange 0,5, Glyzerin 10,0. Am besten wird das Glyzerin erst zugesetzt, wenn beide Farbstoffe im Alkohol gelöst sind. Dauer der Fürbung eine Minute; Differenzieren in 95\% Alkohol, Entwässern, Aufhellen in Ol. Origani, XylolBalsam.

a) Enchondrale Verknöcherung. 'T'ibia.

Die 'libia besitzt bei dem untersuchten menschlichen Embryo aus dem vierten Monat erst einen einzigen Markraum in der liaphyse. Der Knorpel ist bis auf feine Balken aufgelöst, die weiten Markräume sind mit jungem, fast ausschliesslich aus Zellen und Blutgefüssen bestehendem Mark angefüllt: An der Auflösung des Knorpels beteiligen sich zahlreiche mehrkernige Riesenzellen, die den Osteoklasten gleichen; sie liegen zum Teil den Knorpelbalken an, werden aber auch frei in den Markräumen angetroffen. Die meisten Knorpelbälkchen innerhalb des Markraums besitzen bereits einen dünnen Überzug von Knochensubstanz; sie sind durchweg mit Osteoblasten belegt, die in einfacher Lage, einem Epithel ăhnlich, der Oberfläche anliegen.

Die Osteoblasten sind im Vergleich zu den Markzellen als gross zu bezeichnen; sie besitzen ein dichtes Protoplasma, das meistens feinstreifig erscheint. Die Zellen sind prismatisch oder 
zylindrisch, seltener kubisch oder polygonal; der grosse, kugelige Kern liegt meistens endständig und vorwiegend an demjenigen Pol der Zelle, der dem Markraum zugekehrt ist. Natürlich sieht man diese Stellung des Kerns nur bei günstiger Lage der Zelle, wenn ihr längster Durchmesser horizontal gestellt ist, d. h. in der Ebene des Schnittes liegt. Fortsätze sind an den Osteoblasten ziemlich selten; die Streifung des Protoplasmas läuft parallel der Längsachse der Zelle. Wenn nun auch die grosse Mehrzahl der Osteoblasten gleichartig protoplasmatisch erscheint, so kann man an den feinen Knorpelbälkchen nahe der Einschmelzungsgrenze des Knorpels, also an den Enden des Markraumes, einzelne Osteoblasten finden, die von der Mehrzahl abweichen. Thr Zellenleib lässt nämlich $z$ wei deutlich getrennte Abschnitte erkennen. Der eine ist dunkel, protoplasmatisch, enthät den Kern: er ist also meistens dem Knochen abgewendet. An diesen schliesst sich ein ganz heller, hyaliner Abschnitt an, der dem knorpelbitkchenzugewendet ist: er ist of t strukturlos. wie ein Glastropfen, schliesst in anderen Fällen einzelne Körner ein. I)er helle Abschnitt eines Osteoblasten bildet ofters mit dem protoplasmatischen einen Winkel, erscheint wie abgeknickt, so dass er in eine andere Ebene fällt; er ist zuweilen von gleichem Durchmesser wie der protoplasmatische Abschnitt, in anderen Fallen verbreitert er sich und wird zu einem Kegel, dessen verjüngtes Ende mit dem l'rotoplasma zusammenhängt, wahrend die Basis sich an den Knorpelbalken anlegt. Auf der Obertläche eines Knorpelbalkens, oder auch einer dünneren Lage von jungem Knochengewebe, Hiesst der hyaline Abschnitt eines Osteoblasten gleichsam nach allen Seiten hin weiter; er legt sich an die hyalinen Abschnitte benachbarter Osteoblasten an, verschmilzt mit ihnen und bildet eine glasartig aussehende Substanz, welche einen Knorpelbalken oder eine Lage jungen Knochengewebes überzieht. Das ist die erste Anlage der Grundsubstanz des Knochengewebes.

In Fig. 1, 2, 3, Taf. XXI, sind derartige Bildungsstadien der Knochengrundsubstanz von der Tibia eines menschlichen Embryo des vierten Monats wiedergegeben. Die Zeichnungen sind bei $Z$ eiss Apochromat homogene Immersion $2 \mathrm{~mm}$, Komp.-Ok. 6, unter Benutzung des A bbéschen Zeichenapparates 
angefertigt. Es ist besonders Wert darauf gelegt, die Osteoblasten mit ihren beiden Abschnitten, dem protoplasmat is chen und dem byalinen, möglichst naturgetreu wiederzugeben; viele andere Einzelheiten der Präparate sind dagegen nicht dargestellt, wenn sie keine direkte Beziehung zur Bildung der Grundsubstanz des Knochens hatten. Auch die tadellos erhaltenen Mitosen einzelner Warkzellen, die öfters zu sehen waren, sind nicht wiedergegeben.

Die erste Anlage der Grundsubstanz des Knochengewebes auf einem Knorpelbalken im Markraum zeigt Fig. 1. Dem Knorpelbälkchen Kn liegen zwei Osteoblasten an, a und b, deren jeder in zwei Abschnitte zerfällt, einen protoplasmatischen, dunkelu ( $a$ und $b$ ) und einen hellen, hyalinen $\left(a_{1}\right.$ und $b_{1}$ ). Die hyalinen Abschnitte sind die voluminöseren, sie liegen dem Knorpel unmittelbar auf, während die protoplasmatischen nach dem Markraum zu liegen. Nur in b ist der Kern getroffen, der den freien Pol der Zelle einnimmt. Der hyaline Abschnitt ist gegen den protoplasmatischen deutlich abgegrenzt; er liegt nicht genau in der Verlängerung des protoplasmatischen Anteils, sondern erscheint unter leichter Torsion, etwas abgeknickt, so dass die Achsen beider Abschnitte unter stumpfem Winkel zusammentreffen.

Der hyaline Abschnitt eines jeden Osteoblasten verbreitert sich nach dem Knorpel zu, und legt sich auf dessen Oberflache auf. Er scheint sich auf dieser auszubreiten, denn man sieht, dass der Abschnitt $b_{1}$ mit dem ähnlichen Abschnitt $c_{1}$ in Berührung getreten und zu einer Masse zusammengeflossen ist. Dieser hyaline Abschnitt $c_{1}$ ist mit keinem Osteoblasten in Verbindung; es kann aber nach seinem Aussehen keinem Zweifel unterliegen, dass er zu einem Osteoblasten gehört. Zwei Osteoblasten liegen in seiner unmittelbaren Nähe und sind nur durch eine feine Spalte von ibm getrennt (Fig. $1 \mathrm{c}$ ). Es ist möglich, dass der Abschnitt $c_{1}$ von einer dieser Zellen gebildet wurde und sich abgetrennt bat, es kann aber auch sein, dass er von dem zugehörigen protoplasmatischen Abschnitt durch den Schnitt getrennt worden ist. Es handelt sich aber bei den Zellen a und $b$ nicht um einen Sekrettropfen, der noch mit der ihn produzierenden Zelle im Zusammenhang geblieben ist; das ganze Aussehen, die scharfe Begrenzung, der unmittelbare Zusammenhang führt $\mathrm{zu}$ der Erklärung, dass hier Zellen vorliegen, in denen der dem 
Kern ferner liegende Abschnitt des Protoplasmas hell, homogen, glasartig durchsichtig geworden ist. Ein Teil des Zellprotoplasmas hat sich umgewandelt, ist aber noch in Zusammenhang mit dem unveränderten Abschnitt der Zelle geblieben.

Nun besitzt nur eine kleine Anzahl der im Gesichtsfelde befindlichen Osteoblasten den beschriebenen hyalinen Abschnitt; die meisten erscheinen gleichartig und zeigen ein dunkles Protoplasma. Auch ist der byaline Abschnitt nicht leicht zu sehen und kann nur mit starken Systemen -- homogene Immersion erkannt werden. Daraus erklärt sich wohl, dass dieser Abschnitt von fast allen Beobachtern übersehen worden ist. Nur W a ld eye r hat gesehen und beschrieben, dass ein peripherer Abschnitt der Osteoblasten heller wird und in junges Knochengewebe übergeht. Zur Zeit kann diese Angabe genauer gefasst werden. Es handelt sich nicht um eine Bildung, die an der Peripherie des Protoplasma auftritt, und etwa, wie ein Mantel, die Zelle umgibt; sondern es handelt sich um ungefăhr die Hälfte eines Osteoblasten, die dem Knorpel zugekehrt, dem Zellkern abgewandt ist. Diese Halfte wandelt sich in eine helle, homogene Substanz um, bleibt dabei aber noch in Verbindung mit dem dunklen Protoplasma, das dem Kern benachbart liegt.

Wie lange nun die Bildung des hyalinen Abschnitts bei einem Osteoblasten wăhrt, und wie lange dieser Abschnitt mit dem kernhaltigen dunklen Protoplasma in Zusammenhang bleibt, kann nicht genau angegeben werden. Vielleicht erfolgt die Bildung schnell, selten lagenweise, mehr an einzelnen Zellen ablaufend; wenn sich der hyaline Abschnitt kurz nach seinem Entstehen von dem Osteoblasten abtrennt, und sich dann, wie noch geschildert werden soll, weiter umwandelt, so ist erklärlich, warum man die grosse Mehrzahl der Osteoblasten immer als dunkle, einheitlich protoplasmatische Zellen sieht, und nicht auf die Idee kommt, dass es einen Zustand gibt, in dem der Osteoblast Form, Grösse und Aussehen ändert.

In Fig. 2 und Fig. 3, Taf. XXI sehen wir Knorpelbalken, die bereits einen Überzug von jüngsten Knochen besitzen. In Fig. 2 ist der Knorpel eine Strecke weit unterbrochen, und das Bälkchen besteht lediglich aus Knochen, in den schon eine Knochenzelle eingeschlossen ist (Fig. $2 \mathrm{KZ}$ ). Auf den jungen Knochen legen sich, wie anderwärts auf den Knorpel, die 
hyalinen Abschnitte einzelner Osteoblasten auf (Fig. 2, Fig. $3 a_{1}$ ). In Fig. 2 ist der hyaline Abschnitt a gegen den protoplasmatischen a unter nahezu rechtem Winkel abgeknickt; er legt sich mit breiter Fläche dem Knochengewebe an, ist aber noch von diesem zu unterscheiden. (Im Präparat ist der junge Knochen rot gefürbt, der hyaline Abschnitt der Osteoblasten dagegen bleibt farblos, so dass hier die Verschiedenheit beider Substanzen augenfällig ist.) Auch der hyaline Abschnitt des Osteoblasten b, der sich unter dem Osteoblasten a verbirgt (Fig. 2), ist gegen den jungen Knochen gerichtet und legt sich höchst wahrscheinlich an diesen an. Auf diese Weise tragen die Osteoblasten zur Verdickung der jungen Knochenschichte bei; man sieht dies auch in Fig. 3. An zwei Osteoblasten, a und b, ist je ein hyaliner Abschnitt ausgebildet; beide fliessen $\mathrm{zu}$ einer Masse zusammen (Fig. $3 \mathrm{a}_{1}$ ), die mit dem jungen Knochengewebe in Verbindung getreten ist.

Was bedeutet nun der hyaline Abschnitt eines Osteoblasten? Ist er ein Sekrettropfen, oder ist er verändertes Protoplasma? Gegen ein Sekret spricht die Form und die Begrenzung; der hyaline Abschnitt hat eine konstante Lage; die Umrandung des protoplasmatischen Teils der Osteoblasten geht direkt in die des hyalinen Abschnitts über, der Durchmesser desselben ist anfänglich gleich dem Durchmesser des protoplasmatischen Abschnitts, wo die Verbreiterung einsetzt, bleibt die scharfe Begrenzung des hyalinen Abschnitts erhalten - es ist eben die beste Deutung der Befunde die, dass hier ein Stück des Zellenleibes selbst vorliegt, das sich in bestimmter Weise umgewandelt hat. Eine Struktur ist nicht erkennbar, die ganze Masse ist gleichartig, von starker Lichtbrechung. Hier und da findet man ein stark färbbares Korn in die hyaline Masse eingelagert.

Wie nun die Umwandlung des Protoplasmas in den hyalinen Abschnitt erfolgt, habe ich nicht beobachtet. Ich fand den $\mathrm{Ab}$ schnitt gut ausgebildet, oder fand ihn gar nicht vor.

Die Annahme, dass die hyalinen Abschnitte Kunstprodukte seien, ist ausserordentlich unwahrscheinlich. Schon die Tatsache spricht dagegen, dass nur an wenigen Zellen unter den Massen von Osteoblasten der hyaline Abschnitt auftritt. Würde es sich um eine Wirkung eines bestimmten Stoffes auf die Zellen handeln, so müssten doch wohl alle Zellen, die von diesem Stoff beeinflusst 
werden, den hyalinen Abschnitt aufweisen; man würde erwarten müssen, in bestimmten Regionen alle Zellen in dieser Weise verändert zu finden. Man findet aber nur in gut fixierten Objekten die hyalinen Abschnitte der Osteoblasten überhaupt vor. In meinen Präparaten sind alle Mitosen ausgezeichnet zu erkennen; in dem Objekt, dem die Fig. 1 entnommen ist, liegt neben den beiden Osteoblasten c eine Markzelle, die sich gerade geteilt hat; die Knäuelfigur der Tochterkerne ist vortrefflich erhalten. Da darf man folgern, dass die Zellen dieser Gegend gut tixiert sind und dass das, was sie zeigen, natürlichem Verhalten entspricht.

Die Substanz, die sieh durch Zusammenfliessen der hyalinen Abschnitteder Osteoblasten bildet, wird zur Grundsubstanz des Knochengewebes. Die Grundsubstanzdes Knochens ist also umgewandeltes Zellprotoplasma; sie wird lediglich vonden Osteoblastengeliefert.

Ein indirekter Beweis für beide Sitze kanı aus der Anlagerung der hyalinen Massen an die Oberfiache der Knorpelbalken im Markraum, sowie an die die Knorpelbalken umgebende Knochensubstanz entnommen werden. Das einzige, was sich einem Knorpelbalken im Markraum nachweislich auflagert, ist der hyaline Abschnitt der Osteoblasten (Fig. 1).

Da man auf den Knorpelbälkchen nach kurzer Zeit Knochensubstanz antrifft, so liegt der Schluss nahe, dass sich die hyaline Substanz in Knochen umgewandelt habe. Damit wäre auch erklart, warum im jungen Knochen ein Knorpelrest steckt. Nun hat aber der junge Knochen durchaus keine Verbindung mit den Osteoblasten; wenn auch diese Zellen in dichter Reihe dem jungen Knochen aufliegen, so sind sie gegen den Knochen immer deutlich abgegrenzt. Dasselbe gilt von den in die Knochenhöhlen eingeschlossenen Zellen. Diese Tatsache ist aus den Beobachtungen zu erklären. die über das weitere Schicksal der hyalinen Abschnitte der Osteoblasten vorliegen; diese Beobachtungen liefern auch den direkten Beweis dafür, dass sich die hyalinen Abschnitte der Osteoblasten in die Grundsubstanz des Knochens umwandeln. Es trennen sich nämlich die hyalinen Abschnitte von den Osteoblasten und werden zu einem einheitlichen, zusammenhängenden Überzuge für einen Knorpelbalken, oder auch eine Knochenlage; gleichzeitig treten Strukturen in diesem Überzuge auf, die zu 
den Fasern des jungen Knochens werden. Die Bildung eines zusammenhängenden Überzuges für einen Knorpelbalken aus den vereinigten hyalinen Abschnitten benachbarter Osteoblasten zeigt Fig. 4, Taf. XXI. Der Knorpelbalken $\mathrm{Kn}, \mathrm{Kn}_{1}$ wendet das breitere Ende $\mathrm{Kn}$ der Ossifikationsgrenze, das verjüngte Ende $\mathrm{Kn}_{1}$ der Mitte des Varkraumes zu. Während auf diesem bereits eine dünne Hülle von Knochensubstanz gebildet ist (Fig. $4 \mathrm{KS}$ ), liegt näher der Ossifikationsgrenze der Knorpel frei; auf beiden Flächen aber beginnt sich eine homogene Substanz aufzulagern. Auf der konvexen Seite befindet sich der hyaline Abschnitt eines Osteoblasten (Fig. $4 \mathrm{O}_{1}$ ) in Berührung mit dem Knorpel; auf der konkaven Fläche liegt ein ungleich dicker Überzug auf (Fig. 4 $\mathrm{GS}_{\mathrm{r}}, \mathrm{GS}_{2}$ ), der sich zwischen den Knorpel in die Osteoblastenlage $\mathrm{O}_{2}$ einschiebt. Der dickere Abschnitt dieses Überzuges $\mathrm{GS}_{1}$ besteht aus kegelförmigen Massen, die an den Spitzen noch getrennt, mit den Basen aber zusammengeflossen sind; an diese schliesst sich ein dünnerer, gleichmässig entwickelter Abschnitt $G_{\text {S }}$ an, der abgerundet aufhört. Man sieht in dieser homogenen Masse, einzeln wie bei GSt oder zu einer Gruppe vereinigt (GS2), stark gefärbte Körnchen liegen.

Es unterliegt wohl keinem Zweifel, dass dieser hyaline Überzug aus den hyalinen Abschnitten benachbarter Osteoblasten entstanden ist, die sich von den protoplasmatischen Abschnitten der Zellen getrennt haben. Die kegelförmigen Verdickungen (Fig. 4 GSi) reichen bis an zwei Osteoblasten $\mathrm{O}_{2}$ heran; die Spitzen der Kegel werden vom Protoplasma dieser Zellen umfasst. Offenbar ist die Ablösung gerade vollzogen, und daher ist die Kegelform der hyalinen Abschnitte noch zu sehen. Das bestătigt ein Vergleich der Fig. 4 mit Fig. $1, a_{1}$ und $b_{1}$. Die dünnere Abteilung $\mathrm{GS}_{2}$ steht in keiner erkennbaren Beziehung $\mathrm{zu}$ anliegenden Osteoblasten; ich möchte daraus schliessen, dass sie sich schon früher von den zugehörigen Zellen abgetrennt hat. Mit der Trennung ist dann die Möglichkeit gegeben, dass sich die hyaline Substanz gleichmässig auf der Oberfläche des Knorpels ausbreiten kann.

Fig. 11, Taf. XXII zeigt die hyaline, einen zusammenhăngenden Überzug eines Knorpelbalkens bildende Grundsubstanz von der Fläche (Fig. $11 \mathrm{GS}$ ). Der Knorpelbalken $\mathrm{Kn}$ ist teilweise in Auflösung begriffen; in seiner Mitte ist eine grössere Lücke 
entstanden, in der körnige Reste des Knorpels teils frei, teils noch im Zusammenhang mit dem Knorpel zu sehen sind (Fig. $11 \mathrm{Knr}$ ). Die frei gewordenen Knorpelreste bilden grössere und kleinere, aus Körnchen bestehende Schollen ( $\mathrm{Knr}_{1}$ ), welche von der hyalinen Substanz eingeschlossen werden. Die durch die Auflösung des Knorpels gebildete Lücke wird durch die Grundsubstanz völlig ausgefüllt; es erscheint das Knorpelbalkchen sogar an dieser Stelle am dicksten, da die Grundsubstanz in Masse sich angesammelt hat.

Die Körner stellen ein erstes Produkt der Differenzierung vor, die in der Grundsubstanz auftritt, weiter greift und schliesslich zur Sonderung des Kinochens in Grundsubstanz und Fasern fübrt.

Der hyaline, Körnchen einschliessende Überzug des Knorpels muss als "Grundsubstanz" bezeichnet werden; er ist in Wirklichkeit eine, ,homogene und strukturlose Bildung", in der nachweislich alsbald Fasern auftreten. Diese Grundsubstan\% ist nachgewiesenermassen umgewandeltes Zellprotoplasma; sie wird von den Osteoblasten geliefert. Das entspricht genau der von Max Schultze ausgesprochenen Lehre über die Bildung der Grundsubstanzen; und für die Grundsubstan\% des Knochengewebes im speziellen besteht die von $\mathrm{W}$ al deyer stets vertretene Auffassung zu Recht. Noch 1!101 schrieb Walde yer, dass die Grundsubstanz des Knochens „nicht einer Sekretion der Gewebszellen, sondern einer Metamorphose des Protoplasmas derselben ihr Dasein verdanke“. Für diesen Satz liegt nunmehr die Bestätigung in den mitgeteilten Tatsachen vor.

Das Auftreten von Faserzügen in dieser homogenen Grundsubstanz zeigt Fig. $\overline{5}$ und 6 . In Fig. 5 sieht man einen Knorpelbalken $\mathrm{Kn}$ bereits von einer dünnen Knochenlamelle $\mathrm{K} S$ überzogen, die eine Knochenzelle einschliesst. Mit der Knochenlage treten die hyalinen Fortsătze der Osteoblasten b und $\mathrm{c}$ in Verbindung; man sieht, dass die langste Achse des Osteoblasten $b$ und des zugehörigen hyalinen Abschnittes $b_{1}$ parallel der Langsachse des Knochens gerichtet ist. In diesem Fortsatz $b_{1}$ ist eine Längsfaserung leicht angedeutet; ebenso in dem senkrecht $z u b_{1}$ stehenden hyalinen Abschnitt des Osteoblasten c. Körner sind nicht sichtbar. In Fig. 6, Taf. XXI sieht man zwei Osteoblasten $\mathrm{O}_{1}$ und $\mathrm{O}_{2}$ 
annăhernd parallel zur Oberfläche einer Knochenlage gestellt, die den Knorpelrest $\mathrm{Kn}$ einschliesst. In den hyalinen Abschnitten beider Zellen ist ein System von feinen Fasern entwickelt, die bis an die Knochenlage $z u$ verfolgen sind. Der hyaline Abschnitt eines dritten Osteoblasten $\mathrm{O}_{3}$ ist noch homogen, bis auf eine Gruppe feiner Körnchen. Die Fasern sind, wie die Körner, ganz. und gar unabhängig von den protoplasmatischen Abschnitten der Osteoblasten; nur die Grundlage, innerhalb deren sie entstehen, ist Protoplasma; allerdings in bestimmter Weise verändertes Protoplasma.

Die jüngste Knochensubstanz gibt beim Kochen noch keinen Leim; die Fäden der hyalinen Abschnitte der Osteoblasten können demnach ebensowenig als die Fasern in der Knochensubstanz. selbst als ,kollagene Substanz" bezeichnet werden. Auch ist der junge Knochen, im polarisierten Licht untersucht, durchaus is otrop. Es liegen keine Anzeichen dafür vor, dass die Fiaden imnerhalb der hyalinen Abschnitte der Osteoblasten durch Auswachsen aus den Körnern entstehen. Ich möchte annehmen, dass sie direkt aus der Grundsubstanz sich herausdifferenzieren.

Die Osteoblasten bilden also lediglich die Gundsubstanz des Knochengewebes. Fasst man zusammen, was an den Osteoblasten während der Bildung der Grundsubstanz des Knochens vorgeht, so ist folgendes zu konstatieren: Der Osteoblast bildet, meist an dem dem Kern entgegengesetzten Pol der Zelle, einen hyalinen Abschnitt aus, durch Umwandlung eines Teils von seinem Protoplasma. Wihrend der Ausbildung dieses Abschnittes wird die Zelle länger, geht aber nach Trennung von dem hvalinen Abschnitte auf die frühere Grösse zurück. Vor Ausbildung und nach Abstossung des hyalinen Abschnittes sind also die Osteoblasten ron gleichen Dimensionen. Nun war diese Möglichkeit von den Anhängern der Lehre Gegenbaurs nicht in Betracht gezogen; diese setzten voraus, die Osteoblasten müssten kleiner werden, wenn sie die Knochensubstanz aus einem Teil ihres Protoplasmas bilden sollten. Im Gegenteil, ein gewichtiges Argument für die Annahme, dass das Knochengewebe aus einem Sekret der Osteoblasten, und nicht etwa aus umgewandeltem Protoplasma entsteht, wurde aus dem Umstande abgeleitet, dass während der Ausbildung einer Knochenlage die anliegenden Osteoblasten Grösse und Form nicht ändern. So schreibt 
Köllike (Gewebelehre, 6. Aufl., Bd. I, S. 327): „Erstens sind die Ḱnochenzellen in junger, eben erst gebildeter Knochensubstanz oft nicht kleiner als die Osteoblasten; zweitens sind die Entfernungen der Knochenzellen voneinander meist derart, dass man, namentlich unter Berücksichtigung ihrer Grösse, nicht annehmen kamn, dass die Zellen allein die Grundsubstanz des Knochens geliefert haben. Drittens endlich scheiden die Osteoblasten, ohne ihre Gestalt und Grösse zu andern, zuerst eine zellenlose Knochensubstanz ab."

IDie Erklärung dafür, dass die hyalinen Abschnitte der Osteoblasten bisher ïbersehen worden sind, liegt in der Kleinheit und besonders der homogenen Beschaffenheit dieser Bildungen, ferner darin, dass diese Abschnitte nur kurze Zeit mit den Osteoblasten in Zusammenhang bleiben. Man findet auch selten lie hyaline Vorstufe der Knochenlagen auf dell Ḱnorpelbalken ror; meistens sielit man nur die ganz protoplasmatischen Osteoblasten auf dem Knorpel oder auf einer Schichte jungen Kinochengewebes anfliegen. Es folgt wohl daraus, dass das hyaline Stadium der Knochengrundsubstanz nur kurze /eit besteht und (lass es sich schnell auf dem Wege der Faserbildung in junges Knochengewebe umwandelt. Dann ist aber jeglicher '/usammenhang mit den Osteoblasten gelöst. Das gesamte Knochengewebe ist ein Produkt der Osteoblasten; es ist „umgewandeltes Protoplasma". Zuerst ist diese Substanz struktmrlos und sie bleibt strukturlos, so lange sie noch in Zusammenhang mit den Osteoblasten steht. Die Fasern treten erst auf, wenn sich die 'Trennung: von den Osteoblasten vollzogen hat. In diesel Hinsicht kann man sagen, dass die Fasern des Knochengewebes mabhängig vou den Osteoblasten sind. Aber das Material, aus dem die Fasern sich herausdifferenzieren, ist darum doch ein Teil des Protoplasmas der Osteoblasten.

Ob sich die Fasern des jüngsten Knochengewebes direkt in die Fibrillenbündel des späteren linochens umbilden, kann ich nicht sagen; ich habe diese Frage nicht untersucht, da es mir darauf ankam, die Beziehungen des Knochengewebes zu den Osteoblasten aufzuklären. Auch muss ich unentschieden lassen, woher die Osteoblasten selbst kommen und ob sich alle als Knochenzellen erhalten oder ob ein Teil ganz zu Grunde geht. Archiv f. mikrosk. Anat. Bd. 73. 
b) Knochenbildung in Membranen. Belegknochen der Nasenkapsel.

(Fig. 7-12 der Taf. XXI.)

Man könnte vermuten, dass die Beteiligung der Osteoblasten an der Bildung des Knochengewebes am leichtesten bei der Bildung der Knochen in weicher membranöser Grundlage beobachtet werden könne, da hier der einfachste Modus der Knochenbildung vorliegt. Während bei der Knochenbildung im Knorpel zwei Vorgänge gleichzeitig sich abspielen, die Einschmelzung des Knorpels und die Neubildung von Knochengewebe, handelt es sich bei der Knochenbildung in Membranen um eine einfache Gewebsneubildung. Es ist aber in Wirklichkeit viel leichter, die Sonderung der Osteoblasten in den hyalinen und den protoplasmatischen Abschnitt da zu sehen, wo die Osteoblasten sich auf einer knorpeligen Unterlage befinden, als da, wo ihnen eine solche fehlt. Von der hellen Substanz, die zwischen den Zellen des embryonalen Bindegewebes liegt, heben sich die hyalinen Abschnitte der Osteoblasten nicht sonderlich ab und werden sehr leicht übersehen.

Der in membranöser Grundlage auftretende Knochen hat die Form feiner mit Auslaufern besetzter Balken. Ränder und Flächen dieser Balken sind mit Osteoblasten belegt; die Osteoblasten gehen noch eine Strecke weit über die Enden der Knochenbalkchen hinaus, die Richtung andeutend, in der die Bälkchen wachsen werden. Man kann nun, da der neu angelegte Knochen sich in Rubin $S$ intensiv färbt, zwischen den Osteoblasten ein Netzwerk feiner Fäden auffinden, die mit den Knochenbalken zusammenlängen; von einem stärkeren, in der Verlängerung des Knochenbalkens gelegenen Faden gehen nach den Seiten hin, zwischen die Osteoblasten, feine Auslaufer hinein, die sich in dem umgebenden embryonalen Bindegewebe verlieren. Statt der fädigen Ausläufer der Knochenbalken - sie sehen wie Züge von Bindegewebsfasern aus - finden sich auch Reihen gefärbter Körnchen vor; man erhält den Eindruck, als ob die Fäden aus Körnchen sich zusammensetzten. Die Körnchen selbst liegen noch zwischen Osteoblasten.

Auch findet man Fäden und Reihen von Körnern ausser Terbindung mit einem Knochenbälkchen vor; sie wachsen aber 
auf den năchsten Knochenbalken zu. Fig. 12, Taf.XXII zeigt einen Knochenbalken $\mathrm{Kb}$, von Osteoblasten umgeben; bei $\mathrm{b}$ und $\mathrm{c}$ sieht man feine Fuden, die von dem Knochenbälkchen abgehen. Bei a liegen Körner, in Reihen geordnet, sowie feine Fanden, die noch keine Verbindung mit dem Knochenbälkchen haben; bei d sieht man Aggregate von Körnchen auf einem Osteoblasten, bei e ein Geflecht von Fäden, in dem ein Osteoblast steckt.

Es könnte aus derartigen Bildern gefolgert werden, dass die erste Anlage des Knochengewebes eine faserige ist und dass sie zwischen den Osteoblasten, in gewisser Hinsicht unabhängig von diesen Zellen auftritt.

Wenn man nun die feinsten Ausläufer der Knochenbälkchen in dem embryonalen Bindegewebe ausserhalb der Osteoblastenschicht authören sieht, so kann man sie für Bindegewebe halten und dann schliessen, dass die erste Anlage des Knochens aus dem Bindegewebe stammt und dass sie durch Bündel repräsentiert wird, welche unabhängig von den Osteoblasten entstanden sind. Diese Ansicht hat in neuester Zeit v. Korff vertreten. „Die Bindegewebsfibrillen laufen, von verschiedenen Richtungen kommend, pinselartig zusammen; dann legen sie sich zu Fibrillenbündeln aneinander und gehen kontinuierlich in die Fibrillenbündel der ersten Knochengrundsubstanz über" (Die Analogie in der Entwicklung der Knochen- und Zahnbeingrundsubstanz der Säugetiere. Arch. f. mikr. Anatomie, Bd. 69, 1906).

Nun-zeigen derartige Präparate, wie das in Fig. 12 wiedergegebene, wohl die Lage der jungen Knochensubstanz, nicht aber die Entstehungsweise. Diese ist gerade bei der Knochenbildung in Membranen nicht leicht zu sehen; aber sie kann mit Sicherheit erkannt werden. Freilich ist nicht jede Spezies und jedes Alter gleich gut geeignet; mir erwies sich ein menschlicher Embryo von $25 \mathrm{~mm}$ Länge, in Formol-Alkohol fixiert, als ein sehr günstiges Objekt, um die erste Entstehung der Grundsubstanz an den 'Belegknochen der Nasenkapsel zu verfolgen (Fig. 7, 8, 10, 11, Taf. XXI).

Es ist nicht anzunehmen, dass die erste Anlage eines Knochenbälkchens auf andere Weise erfolgt, als die Bildung neuer Substanz an der Oberfläche einer bereits vorhandenen Lage von Knochen. Im Gegenteil, in derselben Weise, in der sich die Anlagerung neuer Grundsubstanz an ein Knochenbalkchen voll- 
zieht, ist sicherlich die erste Bildung von Knochengewebe erfolgt. Der in membranöser Grundlage auftretende Knochen wächst nun dadurch in die Dicke, dass sich amorphe Grundsubstanz auf seiner Oberflache ablagert und zu Knochengewebe wird; er wächst in die Länge, indem neu gebildete Grundsubstanz mit seinen freien Enden in Verbindung tritt. Die Grundsubstanz wird hier, gerade so wie bei der enchondralen Knochenbildung. durch die Osteoblasten gebildet; sie ist umgewandeltes Protoplasma der Osteoblasten.

Zum Belege für diese Angaben mögen die lïg. $\tau, 8,9$, 'Taf. XXI dienen. Fig. 7 und 8 beziehen sich aut ein Knochenbälkchen der Oberkieferanlage vom Menschen; Fig. 8; das freie Ende darstellend, schliesst sich unmittelbar an die in Fig. 7 dargestellte Partie an. l'ig. 9 ist von der Oberkieferanlage eines Schweineembryo entnommen.

Wir sehen in Fig. 7 auf einem Knochenbälkchen $\mathrm{Kb}$ drei Osteoblasten nebeneinander aufliegen $\left(\mathrm{O}_{1}, \mathrm{O}_{2}, \mathrm{O}_{3}\right)$. Jeder dieser ()steoblasten besitzt einen wohl ausgebildeten hyalinen Abschnitt (h1, h.. $h_{3}$ !; auf der Knochenoberfläche beginnen die hyalinen Abschnitte zusammenzufliessen. Der hyaline Abschnitt $h_{3}$ legt sich in ein Grübchen der Oberttäche des Knochens hinein und fïllt dasselbe aus; man sieht den tiefer liegenden Rand des Grübchens deutlich durch die hyaline Masse durchscheinen. Vergleichen wir diesen Befund mit den in Fig. 1, 2, 3, 4 wiedergegebenen Verhältnissen, so liegen offenbar die gleichen Dinge vor. Aus den hyalinen Abschnitten der Osteoblasten bildet sich eine, zunächst strukturlose Auflagerung auf ein Knochenbälkchen, bestimmt, selber $z u$ Knochen $z u$ werden. In Fig. 9 sehen wir gleichfalls, dass auf einer Fläche eines Knochenbälkchens $\mathrm{K}$ sich eine hyaline Auflagerung befindet (Fig. 9 GS); in diese geht der hyaline Abschnitt des Osteoblasten $\mathrm{O}_{1}$ direkt über.

Bemerkenswert ist aber, dass nicht alle Osteoblasten, die einen hyalinen Abschnitt entwickelt zeigen, diesen auf ein Knochenbälkchen auflegen. In Fig. 7 ist der hyaline Abschnitt $h_{1}$ des Osteoblasten $O_{1}$ vom Knochen abgesondert und gegen die bindegewebige Umgebung gerichtet. In einiger Entfernung vom freien Ende des Knochenbalkchens, das in Fig. 7 dargestellt ist, finden wir etwas Ähnliches (Fig. 8). Eine Gruppe von Osteoblasten (Fig. 8 Obl) liegt dem Ende des Knochenbälkchens Kb 
gegenüber; diese Osteoblasten besitzen die hyalinen Abschnitte, die zu einer gemeinsamen Masse h zusammengeflossen sind. Diese Masse liegt im embryonalen Bindegewebe und ist schwierig $\mathrm{zu}$ finden; wenn man aber einmal auf sie aufmerksam geworden ist, erkennt man ihre Natur sehr deutlich. Die hyaline Masse steht durch zwei feine Bälkchen f, f mit den Knochenbälkchen Kb in Verbindung, so dass sie mit ihm eine grosse Lücke L begrenzt, in der zwei Osteoblasten liegen. Der eine von diesen ist vermittelst eines hellen Fortsatzes in Verbindung mit der lyyalinen Masse $h$ getreten.

In dieser Masse selbst erkennt man die Anfünge einer struktur. In der Verlängerung der Osteoblasten sieht man Reihen von Körnchen; den Zwischenräumen zwischen den Osteoblasten entsprechend treten feine Fäden auf. Da diese Veränderungen die gleichen sind, die in den hyalinen Überzügen der Knorpelbalunen zu sehen sind, so handelt es sich hier sicherlich um den gleichen Vorgang. Die aus den hyalinen Abschnitten der Osteoblasten Obl hervorgegangene Masse ist im Begriff, zu Knochen $z u$ werden; sie bildet einen "Herd" von Knochensubstanz in der Umgebung eines Knochenbïlkchens und setzt sich mit diesem in Verbindung.

Schon nach den hier mitgeteilten Befunden muss man als sichergestellt ansehen, dass die Bildung der Grundsubstanz des Knochengewebes innerhalb von Membranen 1. lediglich durch lie Osteoblasten geschieht, und dass sie 2. genau so erfolgt, wie bei der Knochenbildung innerhalb des Knorpels. Die Anlage des Knochengewebes ist hyalin, strukturlos; fadige Bildungen treten erst zu einem bestimmten Zeitpunkte auf, meistens erst, wenn sich die Anlage von den produzierenden Osteoblasten getrennt hat. Wenn man ausgebildete Fasern findet, besteht schon lange kein Zusammenhang mehr zwischen der Knochensubstanz und den Osteoblasten; man darf die Fasern nicht für die erste Anlage des Knocbengewebes halten, sie entsprechen einer ziemlich vorgeschrittenen Stufe der Ausbildung.

Wenn man nun auch erwarten darf, an der Obertlache der Knochenbälkchen, die innerhalb membranöser Grundlage entstanden sind, den Belag mit hyaliner Grundsubstanz zu finden, der auf den Knorpelbälkchen bei der enchondralen Knochenbildung so deutlich zu sehen ist (Fig. 1a, Fig. 4), so muss hervorgehoben 
werden, dass es seine Schwierigkeiten hat, auch mit den besten Systemen die hyaline Grundsubstanz aufzufinden. An den gefärbten und aufgehellten Schnitten bedarf es günstiger Stellen, um die nicht färbbare, glasartig durchsichtige Grundsubstanz des Knochens zu erkennen; am besten sieht man sie noch in den weiten Lücken, die an der Peripherie der Knochenbalken angetroffen werden und Osteoblasten enthalten. Neben diesen Osteoblasten, zuweilen noch im Zusammenhang mit ihnen, ist die neugebildete, strukturlose Grundsubstanz zu sehen. Ich habe versucht, diese für eine Wiedergabe durch die Zeichnung wenig geeigneten Befunde in Fig. 10 und 13 wiederzugeben. Man sieht in Fig. 10 ein Stück "geflechtartigen" Knochens; von dünnen, vielfach verbundenen Balken von Knochensubstanz werden weite Lücken umschlossen. In zweien dieser Lücken liegt hyaline Substanz neben Osteoblasten; in der oben gelegenen Lücke ist deutlich $\mathrm{zu}$ sehen, dass die hyaline Masse $\mathrm{h}$ der helle strukturlose Abschnitt eines Osteoblasten $O_{1}$ ist, dessen Kern nebst der grösseren Menge des protoplasmatischen Abschnittes weggeschnitten ist. In einer angrenzenden Lücke liegt ein Osteoblast $O$; dieserwird von byaliner Substanz $h_{1}$ wie von einer Kappe überzogen. Beim Wechsel der Einstellung tritt der hier verdeckte Rest des Protoplasmas gut hervor. Es hat sich in diesem Falle die hyaline Substanz von dem zugehörigen Osteoblasten abgetrennt; es ist aber nicht zu bestimmen, wo dieser Osteoblast liegt. Nun kann aber wohl keinem Zweifel unterliegen, dass diese, innerhalb von Gruben der Knochenoberfläche befindlichen Massen hyaliner Grundsubstanz sich dem vorhandenen Knochen auflegen, zu Knochen werden und so das Dickenwachstum der Bälkchen fördern. Fig. 13 zeigt ein Stück geflechtartigen Knochens aus der Anlage des Oberkiefers eines menschlichen Embryo; in jeder Lücke liegt ein Osteoblast, der eine hyaline Zone (hZ) entwickelt hat.

Aus den mitgeteilten Beobachtungen ergibt sich, dass zuerst die Grundsubstanz des Knochengewebes gebildet wird. Sie ist ursprünglich homogen, glasartig, strukturlos; sie entsteht aber nicht, wie Gegenbaur lehrt, durch einen Sekretionsvorgang, sondern sie ist "umgewandeltes Protoplasma" der Osteoblasten, wie W ald e y er von jeher ausgesprochen hat. Jeder 
Osteoblast liefert ein Stück Grundsubstanz; dasselbe trennt sich von der produzierenden Zelle, fliesst mit den gleichen Abschnitten benachbarter Osteoblasten zusammen und breitet sich - bei enchondraler Knochenbildung - auf den Knorpelresten innerlaalb der Markräume zu einem zusammenhängenden Überzuge aus. Die zellulare Sonderung ist in diesem umgewandelten Protoplasma endgültig aufgehoben, es ist eine einheitliche Masse geworden, die einen Teil der Zellen, von denen sie abstammt, eingeschlossen hält. Durch Auftreten faseriger Differenzierungen und alsbaldige Erhärtung wandelt sich die Grundsubstanz in junges Knochengewebe um. Man kann diese aus den Osteoblasten entstandene Lage als "Grundsubstanz" oder auch als „osteogenes Gewebe" bezeichnen.

Bei der Knochenbildung in Membranen wird die Grundsubstanz in das embryonale Bindegewebe der Umgebung einfach eingelagert. Ihre Ausdehnung wird durch die Anordnung der Osteoblasten bestimmt. Sowie ein Knochenbälkchen gebildet ist, wächst es durch Anlagerung neu gebildeter Glundsubstanz in die Dicke und in die Länge und verdrangt das Bindegewebe immer mehr.

1)a erst nach der Trennung der Grundsubstanz von den Osteoblasten die fasrige Struktur sich ausbildet - nur ihre erste Andeutung kann schon auftreten, wenn die Grundsubstanz noch mit den Osteoblasten verbunden ist - so sind die Faserzüge des Knochengewebes ausser Verbindung mit den Osteoblasten. Sie sind ausserhalb derselben und unabhängig von ihnen aufgetreten.

Diesel Umstand ändert aber nichts an der Tatsache, dass die Fasern im Knochen Produkte des Zellprotoplasmas sind; unmittelbar haben sie zwar nichts mit den Osteoblasten zu tun; aber mittelbar sind sie auf die Osteoblasten zurückzuführen, da die Grundsubstanz, in der sie gebildet werden, weiter nichts ist, als umgewandeltes Protoplasma der Osteoblasten. Würden die Fasern früber sich ausbilden, so ständen sie in direktem $\mathrm{Zu}$ sammenhang mit Osteoblasten. Man darf also keinen übertriebenen Wert darauf legen, ob man in einem besonderen Falle Fasern innerhalb des Zellenleibes, oder ausserhalb desselben auftreten sieht. Es kommt nur darauf an, zu entscheiden, woher die Grundsubstanz stammt, in welcher die Fasern liegen. Es muss 
gefragt werden : Ist diese Grundsubstanz ein Sekret von bestimmten Zellen, oder ist sie umgewandeltes Protoplasma? In letzterem Falle sind die Fasern in der Grundsubstanz Produlte des Zellprotoplasma, und der Zeitpunkt ihres Auftretens ist von minderer Wichtigkeit. Dieser Gesichtspunkt ist meines Erachtens auch bei den Untersuchungen über die Entstehung der Fasern des fibrillaren Bindegewebes der maßgebende.

Die Resultate der Untersuchungen über die Entstehung des Knochengewebes sind zuerst am 16. Juni 1908 in der Gesellschaft zur Beförderung der gesamten Naturwissenschaften zu Marburg mitgeteilt und in den Sitzungsberichten erschienen. (D is se, Über die Bildung des Knochengewebes. Marburger Sitzungsberichte $1908 \mathrm{Nr}$.5. Juni.)

\section{Die Entstehung des Zahnbeins.}

(Fig. 14-20 auf 'laf. XXII.)

Das Zahnbein bildet sich an der Oberflache einer Zahnpapille, soweit wie diese vom Schmelzorgan bedeckt wird. Die Papille besteht hauptsächlich aus polygonalen Zellen, die zahlreiche, längere und kürzere Ausläufer besitzen und in eine mucinhaltige Grundsubstanz eingelagert sind; vor dem Auftreten des Zahnbeins sind die oberflächlich gelegenen Zellen der Papille durchaus den in der Tiefe gelegenen ahnlich. Sobald nun die Dentinbildung einsetzt, findet man die oberflächliche Zellenlage der Papille in charakteristischer Weise verändert. Sie wandelt sich in eine einfache Schicht hoher, prismatischer Zellen um, deren längster Durchmesser senkrecht zur Oberfläche der Papille steht; man bekommt den Eindruck. als sei die Papille von einem einschichtigen hohen Epithel überzogen.

Das Dentin tritt an der Aussentläche dieser epithelartigen Zellenlage auf, zwischen ibr und dem inneren Epithel des Schmelzorgans; es bildet ein konform der Papillenoberfläche gekrümmtes Scherbchen, das "Zahnscherbchen", das zuerst an der Spitze der Papille auftritt und von dieser aus, an Dicke allmählich abnehmend, auf die Seitenflächen der Papille übergreift. Das Zahnscherbchen reicht nur soweit, wie die erwähnte Lage hoher prismatischer Zellen; es steht mit diesen in Verbindung, indem jede Zelle einen langen feinen Fortsatz in das Zahnscherbchen 
entsendet, für den ein Hohlraum, das Zahnkanälchen, ausgespart bleibt. Die Länge dieses Zellausläufers, der "Zahnfaser", entspricht immer der Dicke der Dentinlage; es nimmt die Zahnfaser in dem Wape an Lünge $z u$, wie die Dentinlage dicker wird.

Wenn man das Zahnscherbchen von der Papille abhebt, lösen sich die hohen, prismatischen Zellen von der Papille ab und bleiben mit dem Zahnscherbchen in Verbindung; sie bilden einen zelligen Überzug an der konkaven Fläche der Dentinlage und sind deshalb als .Dentinzellen" oder "Elfenbeinzellen" benannt worden. Untereinander hängen die Ientinzellen durch kurze Ausliufer zusammen; die meisten lassell einen längeren Ansläufer erkemen, der der Zahnfaser gegenüber von der BasalHäche der Zelle abgeht und in das Grewebe der Papille hinein verläuft.

Diese Befunde führten dazu, dass man die Lage del I)entinzellen als die dentinbildende Schichte ansah. Waldeyer hat diese Auffassung zuerst klar ausgesprochen; er hat die Theorie anfgestellt, dass das /ahubein durch Umwandlung des 'rotoplasmas der Dentinzellen entstehe. An einer jerlen Jentinzelle wandelt sich die Mantelschicht des Protoplasma in die frundsubstanz des Dentins nm, die alsbald durch Einlagerung von Kalksalzen fest wird ; der axiale Protoplasmabschnitt aber bleibt weich, wird zur Zahnfaser und bleibt natürlich in dem neugebildeten Dentin liegen. Bei dieser Umwandlungsweise muss nun die Dentinzelle bald verbraucht werden: ein T'eil des Protoplasma wird zu Dentin, ein anderer zur Zahnfaser. Dann tritt eine neue aus der P'apille stammende Zelle für die verbranchte ein. „Durch den Pulpafortsatz steht jeder Odontoblast mit den tiefer gelegenen, sich ebenfalls sukzessive vergrössernden Zellen der jungen Pulpa (d. h. der Papille) in Verbindung, sodass, wenn ein Odontoblast bis auf das Faserrudiment verknöchert ist, ein anderer an seine Stelle tritt, ohne dass die Kontinuität der laser unterbrochen wird. Dem Gresagten zufolge muss also jede Zahnfaser als ein Rudiment mehrerer zusammenhängender Odontoblasten angesehen werden ... Somit konstituiert sich das Dentin mit allen seinen Bestandteilen nur aus den chemisch und formell umgewandelten Odontoblasten." (Waldeyer, Bau und Entwicklung der Zähne. Strickers Handbuch der Lehre von den Geweben, Bd. I, 1871.) 
Der Kernpunkt der Lehre Waldeyers ist der, dass das Dentin umgewandeltes Tellprotoplasma ist. Die Einzelheiten des Umwandlungsprozesses aber waren von $\mathrm{W}$ ald eyer weniger direkt gesehen, als vielmehr erschlossen worden. Besonders gilt dies für die Angabe, dass die Odontoblasten ganz zur Bildung von Dentin und Zalınfaser verbraucht würden, und dass immer neue Odontoblasten entstehen und an die Stelle der verbrauchten treten sollten. Gegen diesen Punkt richteten sich zunächst die Einwürfe. Kölliker (Gewebelehre, 5. Aufl., 1867) sprach sich dahiin aus, „dass in vielen Fallen eine einzige Zelle ausreicht, um eine Zahnfaser zu bilden: man sieht an den Zahnfasern nie Spuren einer Entstehung aus Zellreihen". Vielmehr nahm Kölliker an, "dass die Elfenbeinzellen, indem sie einerseits immer neuen Bildungsstoff aufnehmen und hierdurch in immer gleicher Grösse sich erhalten, auf der andern Seite durch ein lebhaftes Spitzenwachstum immer längere verästelte Ausläufer, eben die Zahıfasern, hervorbringen". Damit war der neue Gesichtspunkt eingeführt, dass die Dentinzelle ständig in die Lünge wachsen kam, und dass sie dadurch etwaigen Verlust an I'rotoplasma zu ersetzen imstande ist. Indessen war Kölliker der Meinung, dass das Wachstum lediglich den Verlust desjenigen Protoplasmaabschnittes decke, der zur Bildung del Zahntaser verbraucht werde. Eine Umwandlung des Protoplasmas einer Dentinzelle in Dentin komme nicht vor; es handle sich vielmehr um eine A usscheidung, die von den Dentinzellen geliefert wird, verkalkt und zu Dentin wird. Weil das Dentin zwischen den äusseren Enden der Dentinzellen auftritt, "geht es nicht wohl an, das Dentin unmittelbar aus der Pulpa abzuleiten, und es bleibt nichts anderes übrig, als anzunehmen, dass das Dentin unter Vermittlung der Elfenbeinzellen sich bilde". (Grewebelehre, S. 387.) Die Grundsubstanz des Dentins wäre "als eine durch alle Elfenbeinzellen gemeinsam gebildete Ausscheidung zu betrachten"; diese ist durchaus gleichartig und lässt niemals eine Zusammensetzung aus einzelnen Abschnitten erkennen, die man etwa für Zellterritorien halten könnte.

Kölliker zog noch eine scharfe Grenze zwischen einer Sekretion und einer Umwandlung des Zellprotoplasma; es lag wohl die Vorstellung zugrunde, dass ein Sekret immer amorph, vielfach sogar tlüssig sei, während dem sich um- 
wandelnden Protoplasma immer eine Struktur zukomme; dass ferner ein Sekret sich von den produzierenden Zellen sofort trenne, während Protoplasma mit ihnen im Zusammenhang bleibe. Wenn man zulässt, dass Protoplasma seine Struktur verlieren, amorph werden und sich aus dem Zellverbande lösen kann, so wird Protoplasma zu Sekret; es verwischt sich die Grenze zwischen beiden Vorgängen, da das Produkt in dem einen wie in dem anderen Falle "umgewandeltes Protoplasma" ist. In diesem Sinne hat v. Ebner (Köllikers Gewebelehre, 6. Aufl., 1899) die Lehre Köllikers über die Entstehung des Dentins modifiziert; er hält es den Tatsachen am meisten entsprechend, anzunehmen, „dass die Elfenbeinzellen an ihrer Oberfläche zunächst eine nicht fibrilläre Substanz bilden, die zu einer gemeinsamen Masse zusammen fliesst" . . „Die fibrilläre Grundsubstanz des Zahnbeins wird von den Elfenbeinzellen zunichst an der ausseren Oberfläche, dann aber auch zum Teil an den sich berührenden Seitenflächen der Zellen gebildet." Denn das zuerst gebildete Dentin erscheint "wie ein Ausguss der Zwischenrüume zwischen den äusseren Enden der Dentinzellen"; es entsteht nauf Kosten der Odontoblasten wahrscheinlich dadurch, dass die Plasmafäden derselben an der Oberfläche zu einer chemisch dem Kollagen nabe stehenden Substanz, die zunächst nicht fibrillarr ist, zerfliessen" (v. Ebner, Über die Entwicklung der leimgebenden Fibrillen, besonders im Zahnbein. Wiener Sitzungsberichte, math.-naturw. Kl., Bd. 115, Abt. 3, 1906).

Bei dieser Auffassung bleibt aber die Lehre von Kölliker bestehen, dass das Zahnbein das Produkt einer einzigen Lage von Dentinzellen ist. Die Zellen wachsen, solange sie Dentin produzieren, und ihr nicht zu Dentin gewordenes Protoplasma erhält sich in Form der Zahnfasern. Nur in diesem Punkte entfernt sich die Lehre v. Ebners von der Theorie, die Wald ey er aufgestellt hatte; sie fasst aber, dieser Theorie entsprechend, das Dentin als umgewandeltes Protoplasma auf. Der Umwandlungsprozess ist aber auch von v. Ebner nicht in seinen Einzelheiten gesehen; besonders sind keine Beobachtungen mitgeteilt, die eine sichtbare Verănderung des Protoplasmas der Dentinzellen bei der Dentinbildung feststellen.

Nach den zitierten Autoren, denen eine Anzahl anderer anzureihen wäre, sind es immer die Dentinzellen, von denen die Bildung des Dentins ausgeht. 
Nun ist in neuester Zeit angegeben worden, dass das junge Dentin, das "wie ein Ausguss" zwischen den ausseren Enden der Dentinzellen liegt, nicht homogen sei, sondern fibrillär; dass die Fibrillen aus der Papille herkommen, und durch Auffaserung stärkerer Bündel entstehen, die zwischen den Dentinzellen hindurchtreten. Diese Fibrillen werden direkt zu den Fibrillen des fertigen Zahnbeins. Es ist keine anfangs homogene Grundsubstan\% des Dentins da, sondern die erste Anlage desselben ist fibrillär; die Fibrillen stammen aus dem Gewebe der Papille. Iie Zahnbeingrundsubstanz „baut sich aus Fibrillen auf, die mit denen der Zahnpulpa kontinuierlich sind". (v. Korff, Die Entwicklung der Zahnbeingrundsubstanz der Säugetiere. Arch. f. mikr. Anatomie, Bd. 67, 1905.) Wenn diese Angaben zutreffen, ist das Dentin ein Produkt der Zahnpapille, und zwar ihrer Fasern, nicht ihrer Zellen; die Dentinzellen kommen dann für die Bildung des '/ahnbeins nicht in Betracht.

Nach meinen eigenen Untersuchungen, die hauptsïchlich an menschlichen Fmbryonen angestellt sind, ist es nun möglich, den Nachweis zu führen, dass die Dentinzellen es sind, welche das Zahnbein produzieren. Das Dentin ist umgewandeltes \%ellprotoplasma der Dentinzellen; die einzelnen Umwandlungsstadien lassen sich erkennen, und sie entsprechen im wesentlichen den Veranderungen, die das Protoplasma der Osteoblasten bei der Bildung des Knochengewebes erführt.

Das Verständnis für die Vorgänge bei der Bildung des Lentins erschliesst sich nur dem, der die Bildung der Knochensubstanz in ihren Einzelheiten untersucht hat, und der besonders die Umbildungen der Osteoblasten, das Homogenwerden des peripheren Zellellabschnitts und seine Trennung von dem protoplasmatischen, genau kennt. Diese Vorgänge sind an den Osteoblasten relativ leicht $\mathrm{zu}$ sehen; ihre Deutung ist nicht schwierig, and sie erleichtert das Verständnis anologer Vorgänge in verwandten Geweben. Die Veränderungen an den Dentinzellen sind schwieriger zu sehen; man wird nur dann auf ihre Bedeutung aufmerksam, wenn man die Phasen kennt, die ein Osteoblast bei der Produktion von Knochengewebe durchläuft. Man wird immer wieder die Wahrheit des Satzes bestătigt finden, den vor Jahren Wal d eyer aussprach: "Die histiologische Genese des Zahnbeins muss voll- 
kommen der Bildung der Knochensubstanz homologisiert werden" (Strickers Handbuch, Bd. I, S. 350).

Auch zur Untersuchung der Dentinbildung dienten menschliche Embryonen, die teils in Pikrinsäure-Sublimat, teils in Zenkerscher Lösung fixiert waren. Es kamen Embryonen von $125 \mathrm{~mm}, 132 \mathrm{~mm}, 140 \mathrm{~mm}$ Länge zur Verwendung, ausserdem ein Embryo aus dem Ende des 5. Monats, der in Müllerscher Lösung fixiert worden war. Auch wurden Schweineembryonen, in Sublimat-Eisessig fixiert, zum Studium der Ientinbildung herangezogen. Die Vorbehandlung aller Prüparate war die folgende: Entkalkung in $10 \%$ Kochsalzlösung mit $2 \% \mathrm{HCl}$; Stückfärbung in Hämalaun, Einschluss in Paraffin. Die 0,005 mm dicken Schnitte wurden auf dem Objektträger eine Minute lang in einer alkoholischen Lösung von Rubin S und Orange nachgefärbt (Rubin S 1,0, Orange 0,5, 95 0 Alkohol 90,0, Glyzerin 10,0), in starkem Alkohol differenziert und in Ol. Origani aufgehellt. Bei dieser Behandlung werden die Zellkerne blau, das Protoplasma der Dentinzellen rot; das jüngste Dentin erscheint rot, das iltere olange. Die /ahlnscheiden werden rot. Die Fragestellung muss lauten: Kommen wälrend der Dentinbildung an den Dentinzellen sichtbare Veranderungen vor? Haben diese Veranderungen Ïhnlichkeit mit denen, die an den Osteoblasten bei der Knochenbildung beobachtet werden? Liefern die veränderten Abschnitte der Dentinzellen das junge Dentin? Üm diese Fragen beantworten zn können, müssen wir mit der Schilderung der Dentinzellen beginnen.

Um die Zeit, in welcher die Dentinbildung beginnt, tritt auf der Obertläche der Zahnpapille eine Lage bestimmt geformter Zellen auf, die sich von den zentralen Zellen der Papille unterscheiden. Es sind die "Dentinzellen", auch „Odontoblasten“, oder „Elfenbeinzellen" genannt. Die Schichte dieser Zellen ist anfänglich, bei ihrem ersten Auftreten, am dicksten, und nimmt allmählich an Mächtigkeit ab. Man findet 3-5 Zellenlagen übereinander, besonders an der Spitze der Papille, während in späterer Zeit höchstens zwei Zellenlagen da sind. Die einzelnen Zellen sind zwar an Form, Grösse, Zahl der Ausläufer vielfach verschieden; indessen treten die Abweichungen gegenüber den gemeinsamen Merkmalen zurück. Die Dentinzellen sind lange und schmale, prismatisch oder auch zylindrisch gestaltete Zellen. Der Kern liegt, mit seltenen Ausnahmen, endständig, in dem- 
jenigen Ende der Zelle, das der Papille zugekehrt ist; man kann es als das zentrale Ende bezeichnen. Der Kern ist gewöhnlich kuglig, seltener ovoid; er hat eine deutliche Struktur, das Chromatin bildet ein feines Netzwerk mit zahlreichen grösseren und kleineren Netzknoten. Das Zellprotoplasma ist undeutlich streifig, die Streifen laufen der Längsachse der Zelle parallel. Ausser den feinen Fibrillen, die die Streifung bewirken, enthält das Protoplasma zahlreiche feine Körner; wenn die Streifung zurücktritt, kann die Zelle körnig erscheinen.

Die Länge der Dentinzellen beträgt nach Walde yer beim Menschen 20-30 $\mu$, die Breite $5 \mu$; Kölliker gibt 35-54 $\mu$ Lange bei $5-10 \mu$ Breite an. Ich finde bei menschlichen Embryonen die Länge $24-30 \mu$, die Breite $5-6 \mu$. Der Kern hat den gleichen Durchmesser wie die Zelle.

Es ist unter Umständen nicht leicht, die Länge einer Dentinzelle genau zu bestimmen; einmal besitzt die Zelle Fortsïtze, auch solche, die in der Richtung des lingsten Durchmessers liegen, nämlich die Zahnfasel und einen ilır gegenüber abgehenden zentralen Fortsatz. Wenn man ein kleines Stück dieser Fortsittze bei der Messung berücksichtigt, bekommt man natürlich liöhere Werte, als wenn man die Fortsätze ausser acht lässt. Wichtiger aber ist die Tatsache, dass der leicht sichtbare, protoplasmatische Abschnitt einer Dentinzelle sebr häufig nicht die ganze Zelle darstellt, sondern nur einen Teil derselben.

Es ist mehrfach angegeben worden, dass die Dentinzelle, wenn die Bildung des Dentins im Gange ist, in zwei Zonen zerfällt. v. Korff sagt: „der Zellenleib der Elfenbeinzelle lässt einen kleinen äusseren, homogenen, und einen grösseren, inneren, differenzierten Abschnitt erkennen "; v. E b n er bildet derartige in zwei Zonen gesonderte Dentinzellen ab (Köllikers Handbuch der Gewebelehre, 6. Aufl., Bd. 3, Fig. 935).

Die Beschreibungen und die Abbildungen der genannten Autoren beziehen sich auf isolierte Zellen, und diese zeigen die ausseren, homogenen Abschnitte der Dentinzellen nur sehr unvollstăndig. Man muss die Zellen an Schnittpräparaten untersuchen, wenn man das wirkliche Verbalten der homogenen Zone erkennen will. Es ergibt sich dann, dass die Dentinzelle vielfach in eine innere oder zentrale protoplasmatische, und in eine äussere oder periphere hom $\mathrm{og}$ e $\mathrm{n}$ e Zone zerfallt, und dass 
die homogene Zone ein variables Element ist. Das Auftreten dieser homogenen Zone bängt nun mit der Bildung des Dentins zusammen, es stellt das erste Stadium bei der Dentinbildung dar.

Die Bildung des Dentins wird dadurch eingeleitet, dass die Dentinzellen einen homogenen, nach aussen gekehrten Abschnitt ausbilden, der dem Schmelzorgan anliegt. Dieser Abschnitt wird glasartig durchsichtig, besitzt keine deutliche Struktur, nimmt an Durchmesser gegenüber dem protoplasmatisch bleibenden $\mathrm{Ab}$ schnitte der Zelle $z$, und tritt mit den gleichartig veränderten ausseren Abschnitten der benachbarten Dentinzellen in innige Berührung. Dann Hiessen die in Verbindung stehenden Enden der homogenen Abschnitte an der inneren Grenze des Schmelzorgans zu einer einzigen Lage zusammen. Diese Lage, hervorgegangen aus dem in bestimmter Weise veranderten Protoplasma der Dentinzellen, trennt sich von den Dentinzellen ab und wird zu Dentin. Das Dentin wächst in die Dicke durch Anlagerung neu gebildeten Materials an die bereits vorhandene Dentinschicht; dieses Material geht auf die gleiche Weise, wie das zuerst gebildete Dentin, ilus den Dentinzellen hervor.

Fig. 14, Taf.XXII zeigt das erste Stadium der Dentinbildung; in Fig. 14a sehen wir die Verdickung einer bereits vorbandenen Dentinschicht durch Anlagerung einer hellen, noch mit den Dentinzellen in Verbindung stehenden Lage. Beide Präparate gehören einer Serie an, die von einem Schweineembryo stammt.

Fig. 14 zeigt die erste Bildung derjenigen Substanz, die zu Dentin werden soll; man kann sie mit v. Ebner als "Prädentin" benennen. Die Stelle, die in der Abbildung wiedergegeben ist, liegt ganz nahe dem zugeschärften Rande des Zahnscherbchens. Es ist noch kein Dentin da, wohl aber eine gut ausgeprägte Lage von Dentinzellen. Diese erscheint bei schwächerer Vergrösserung von dem inneren Epithel des Schmelzorgans durch einen hellen Zwischenraum getrennt; die Untersuchung mit homogener Immersion aber zeigt, dass die helle Partie aus den ganz hellen, gleichmässig hyalin erscheinenden Abschnitten der Dentinzellen besteht (Fig. $14 \mathrm{hZ}$ ). Eine jede Dentinzelle besteht aus zwei Abschnitten, einem protoplasmatischen ( $\mathrm{p} Z$ ), der an die Papille angrenzt und den Kern einschliesst, und einem hellen, glasartig durchsichtigen (hZ), der unter leichter Verbreiterung aus dem protoplasmatischen Abschnitt hervorgeht und sich mit 
seiner ausseren Fläche an das Schmelzorgan (SO) anlegt. In der Năhe des protoplasmatischen Zellenabschnittes sind die glasartigen Partien selbständig; ihre breiten Aussentlächen aber sind entlang dem Schmelzorgan miteinander verschmolzen und bilden eine einzige, gleichmässige helle Lage, die mit dem Rande des Zahnscherbchens in Berührung steht.

Ein ganz gleiches Verhalten zeigen die Dentinzellen an der Innentiache des 7ahnscherbchens, also näher der Spitze der Zahnpapille (Fig. 14 a, Taf. XXII). Die Dentinlage (D) besitzt eine gewisse Mächtigkeit, 30-40 "'; sie besteht aus einer ausseren, gleichartigen und einer inneren \%one, die sich in Rubin stark gefärbt hat. In dieser Zone sind die Zalmkanälchen deutlich wahrzunehmen; die Grundsubstanz erscheint körnig und enthält noch keine anisotropen Fibrillenzüge. Es ist also ganz junges Dentin Unterhalb dieser Schichte ist eine ganz helle, homogene Lage $h \%$ vorhanden; sie setzt sich aus den ausseren, hellen und homogenen Abschnittell der Dentinzellen zusammen. so dass alle diese Zelien mit der genannten Schicht in Verbindung stehen. bie hellen Aussenzonen der Dentinzellen sind von ungleicher Länge; sie sind zylindrisch, oder auch kegelförmig. In der hellen Substanz treten bei einzelnen Zellen Körnchen auf. die sich in Rubin stark färben; sie liegen immer ganz obertlächlich. stehen einzeln oder ordnen sich zu Reihen zusammen. Auch die Grenze des protoplasmatischen Abschnittes der Dentinzellen gegen den hellen Abschnitt kann körnig erscheinen.

Die Dentinzellen, die sich in zwei Abschnitte gesondert haben, gleichen durchaus den Osteoblasten, die einen Teil ihres Protoplasmas in osteogene Substanz umwandeln; es kann keinem Zweifel unterliegen, dass in beiden Zellenarten analoge Vorgänge sich vollziehen; die darauf hinauslaufen, eine Substanz zu bilden, welche später sklerosieren soll.

Die Dentinzellen des Menschen lassen die beim Schweineembryo beschriebenen Stadien gleichfalls erkennen. Es wird der nach aussen gerichtete Abschnitt des Zellprotoplasmas in eine helle, homogene Masse verwandelt, die als ein peripherer $A b$ schnitt der Zelle erscheint. Fig. 15, Taf. XXII zeigt vier derartig veränderte Dentinzellen, die an der Innentläche einer dünnen Schichte von Dentin (D) gelegen sind. Der protoplasmatische Zellenabschnitt (p Z), der an seinem zentralen Ende den 
Kern einschliesst, geht obne scharfe Grenze, mehr allmahlich, in den homogenen Abschnitt $\mathrm{h} \mathrm{Z}$ über; die sumtlichen homogenen Abschnitte aber sind bereits miteinander verbunden und zu einer einzigen Masse zusammengeflossen, die sich enge an die Dentinlage D anschliesst. Auch in Fig. 16, Taf. XXII sieht man die hyalinen, homogenen Abscbnitte von drei Dentinzellen miteinander verschmolzen und zu einer glasartig hellen Masse $\mathrm{h} Z$ vereinigt. Es ist die Spitze der Papille eines Schneidezahns bei einem Embryo von $132 \mathrm{~mm}$ Länge dargestellt, auf der sich das Zahnscherbchen $D$ gerade anlegt. Die Grenze beider Abschnitte erscheint an den Dentinzellen unregelmässig; an der mit $\mathrm{p} Z$ bezeichneten Zelle entsendet der protoplasmatische Abschnitt zwei Auslâufer in die hyaline Masse hinein; nur die mit $\mathrm{p} Z_{1}$ bezeichnete Zelle zeigt eine geradlinige Abgrenzung beider Abteilungen.

Überall da, wo sich Dentin bildet, nimmt man das Auftreten einer hyalinen Aussenzone an den Dentinzellen wahr. Es muss diese Veränderung eine wesentliche Bedeutung haben. Dass sie bisher gar nicht oder nur unvollkommen gesehen worden ist, liegt wohl an der Durchsichtigkeit des hyalinen ausseren $\mathrm{Ab}$ schnittes; oft kann man geneigt sein, ihn für eine Lücke im Präparat zu halten. Es bedarf einiger Erfahrung und einiger Aufmerksamkeit, um die bellen Abschnitte der Dentinzellen zu sehen; um die richtige Deutung der Bilder geben zu können, ist es notwendig, die Verănderungen der Osteoblasten bei der Knochenbildung zu kennen. Nicht selten sieht man, besonders auf Flächenschnitten der Dentinzellen, glasartige Massen zwischen den Zellen liegen, deren Zusammenlang mit protoplasmatischen Abteilungen nicht nachzuweisen ist. Derartige Bilder erklären sich daraus, dass oftmals die beiden Abschnitte einer Zelle einen Winkel miteinander bilden, sodass der Schnitt nur einen einzigen Abschnitt trifft. Vielfach kann man aber auch den Nachweis führen, dass glasartige Massen mit kernhaltigem Protoplasma zusammenhängen. Nun ist die wichtige Frage zu beantworten: In welcher Weise wandelt sich die homogene, aus den äusseren Abschnitten der Dentinzellen gebildete Lage weiter um? Welcherlei Stadien durchläuft sie, bis sie $z u$ Dentin geworden ist?

Die homogene Lage enthalt zweierlei Bestandteile: einmal das Material, das zu Dentin wird, ferner die Zahnfasern. Beiderlei 
Elemente lassen sich anfanglich, so lange die hyalinen Abschnitte noch mit den Dentinzellen vereinigt sind, nicht voneinander unterscheiden. Die ganze, den Dentinzellen aufsitzende Lage ist homogen; das sieht man sehr gut an dem in Fig. 17, Taf. XXII wiedergegebenen Langsschnitt der Zahnanlage eines menschlichen Embryo von $140 \mathrm{~mm}$ Lange. Die Dentinlage D wird durch die homogene Schicht $\mathrm{hZ}$ von den Dentinzellen getrennt; die Schicht $\mathrm{hZ}$ setzt sich aus Abteilungen zusammen, deren jede zu einer Dentinzelle gehört, was auf den ersten Blick erkennbar ist.

Nun trennt sich die homogene Lage von dem protoplasmatischen Abschnitt der Dentinzellen ab und wird selbstindig; gleichzeitig werden in ihr die Zahnfasern sichtbar, die mit den Dentinzellen in Verbindung stehen. In Fig. 18, Taf. XXII hat fast eine jede der Dentinzellen $\mathrm{DZ}$ einen langen peripheren Fortsatz Zf, der in ein Kanclchen des Dentins D binein verfolgt werden kann. Es ist die Zahnfaser. Diese besitzt jetzt dic Struktur und die Farbbarkeit des protoplasmatischen Abschnittes der Dentinzelle; vorher, so lange die homogenen Abschnitte noch mit den protoplasmatischen in Verbindung stehen, ist nichts von einer Faser zu sehen, es hebt sich innerhalb des hyalinen Abschnittes einer Zelle das in der Achse gelegene Protoplasna nicht gegenüber dem peripheren durch besondere Lichtbrechung hervor. Indessen kann wohl keinem Zweifel unterliegen, dass die Zahnfaser innerhalb des homogenen Abschnittes einer Dentinzelle von Anfang an vorhanden ist, wenn sie auch anfänglich noch dasselbe Lichtbrechungsvermögen hat wie das umgebende, hyalin gewordene Protoplasma.

Von dem Zeitpunkt ab, in welchem die hyalinen Abschnitte der Dentinzellen sich von den protoplasmatischen trennen, bleibt die Zahnfaser sichtbar, und erscheint als ein Auslaufer des protoplasmatischen Abschnittes der Zelle. Natürlich steckt eine jede Zahnfaser in der homogenen Masse, die aus den hyalinen Abschnitten besteht; wir wollen sie von jetzt ab als "Pradentin“ bezeichnen (Fig. 18 PD).

Es sind zuerst von $H \ddot{b l}$ l (Beitrag zur Histologie der Pulpa und des Dentins. Arch. f. Anatomie 1896) Fasern beschrieben worden, die aus dem Gewebe der Zahnpapille kommen, die Lage der Dentinzellen durchsetzen und bis an die innere Fläche des Schmelzepithels herantreten Die Fasern lösen sich $z$ wischen den 
Alusseren, peripheren Abschnitten der Dentinzellen in feinste Fibrillen auf. v. Korff bat diese Fasern und die aus ihnen hervorgehenden Fibrillenzüge ebenfalls gefunden und sie für die Vorläufer der Fibrillen des Dentins erklär. Wenn nun auch diese Deutung nicht aufrecht zu halten ist, weil die fraglichen Fasern senkrecht zu der Verlaufsrichtung der Dentinfibrillen stehen und weil sie weder leimgebend noch doppeltbrechend sind (v. Ebner), so ist die Existenz dieser Fasern, die sich in Fibrillen auflösen, nicht $\mathrm{zu}$ bestreiten. Wenn die Dentinbildung im Gange ist, kann man diese "HöhIschen Fasern" oftmals nachweisen. Sie gehören zum Gewebe der Zahnpapille und sind teils Zellenauslaufer, teils Fasern, die in einer "Grundsubstanz" differenziert sind und mit den Zellen der Papille keinen nachweisbaren Zusammenhang mehr besitzen. Wo bleiben diese Fasern bei der Dentinbildung? Höhl bildet ihren Übergang in das junge Dentin $a b$; das junge Dentin liegt aber nach seiner Zeichnung $z$ wischen den peripheren Enden der Dentinzellen, gan\% ahnlich sind die Abbildungen, die v. Korff gibt.

Beide Autoren haben nicht erkannt, dass die jüngste Dentinlage aus den homogenen, verbreiterten und zusammengeflossenen Abschnitten der Dentinzellen besteht; dass sie nicht wie ein Ausguss $z$ wischen den Dentinzellen liegt, sondern dass sie eine direkte Verbindung mit den protoplasmatischen Abschnitten dieser Zellen besitzt, also zu den Dentinzellen gehört. Bei dem $\mathrm{Zu}$ sammentliessen der homogenen Abschnitte der Dentinzellen zu einer einzigen Lage kommen die Fasern, die zwischen den Dentinzellen gelegen waren, in die homogene Masse, also in das Pradentin, hinein zu liegen; sie werden vom Prädentin eingeschlossen. Eine Zeitlang bleiben die Fasern noch sichtbar; auch färberisch lassen sie sich darstellen. Wenn aber die Umwandlung des Pradentin in Dentin eintritt, verschwinden die genannten Fasern völlig. Sie verhalten sich $z u$ dem Dentin, wie die Knorpelbalken innerbalb der Markräume sich zum jungen Knochengewebe verhalten; auch diese werden allseitig vom neugebildeten Knochen umgeben, bleiben kurze Zeit sichtbar, können farberisch nachgewiesen werden, sind aber bei einem bestimmten Alter eines Knochenbalkens nicht mehr nachweisbar. Sie werden dem umgebenden Gewebe assimiliert. Die so viel dünneren Fasern im Dentin werden von dieser Assimilation noch 
früher betroffen als die Knorpelreste innerhalb des jungen Knochens.

Wenn das Prädentin zu einer zusammenhängenden Schicht geworden ist, die sich von dem Rest der Dentinzellen abgetrennt hat, ist es zu einer "Grundsubstanz" geworden. In dieser sind aber keine zelligen Elemente enthalten; da die Grundsubstanz nur an einem Pol der sie produzierenden Zellenlage entsteht, bleiben die Mutterzellen - also die Dentinzellen - an der inneren Fläche der Grundsubstanzlage liegen und nur ein Rest des Protoplasma einer jeden Dentinzelle, der nicht in Grundsubstanz umgewandelt worden ist, bleibt als "Zahnfaser" in der Schicht liegen. Für jede Zahnfaser bleibt ein relativ weiter Hohlraum ausgespart; vielleicht enthält er, ausser der Faser, noch Flüssigkeit.

Das Prädentin wird fester, härter, bildet bestimmt orientierte Fibrillenzüge aus und verkalkt dabei. Die "Sklerosierung" des Prädentins wird nun von sichtbaren Veränderungen begleitet. In der gleichartigen Masse tritt eine Art von provisorischer Struktur auf; es bilden sich nämlich feine, in Rubin $S$ färbbare Körner, die bis zum Auftreten der Fibrillenzüge sichtbar bleiben. Die Bildung der Körner beginnt am inneren Rande des Prädentins, also in der unmittelbaren Nähe der protoplasmatisch bleibenden Zellenabschnitte, die von nun an die ganzen Dentinzellen repräsentieren und schreitet nach aussen nach dem Schmelzorgan hin, vor; in der unmittelbaren Nähe der Lage fertigen Dentins sieht man öfters noch körnerfreie, glasartig durchsichtige Massen von Prädentin, die man leicht für Lücken der Substanz balten kann (Fig. $19 \mathrm{HZ}$ ).

In der körnig gewordenen Masse des Prädentins sind an feinen Schnitten die Kanäle für die Zahnfasern gut zu erkennen (Fig. $19 \mathrm{R}$ ). Der Schnitt nimmt mitunter die Dentinzellen und ibre Auslaufer weg, so dass die Raume, in denen sie gelegen haben, sichtbal werden. Meistens aber bleiben die Dentinzellen mit ihren peripheren Enden im Prädentin stecken (Fig. $19 \mathrm{DZ}$ ). Man kann aus dieser Erscheinung entnehmen, dass das Prädentin an Festigkeit gewonnen hat. Die Hohlrăume des Prădentins stehen, wie man vielfach sehen kann, in Verbindung mit den Kanalchen der fertigen Dentinschicht; sie bilden gleichsam etwas erweiterte, leicht schraubenförmig verlaufende Vorrăume dieser Kanälchen. 
Wenn die ganze Lage von Pradentin lörnig und fest geworden ist, tritt sie in Verbindung mit der fertigen Dentinschicht und wandelt sich dann erst in Dentin um. Der Untersclied zwischen Prädentin und Dentin liegt darin, dass im Dentin die Bündel leimgebender Fibrillen, parallel der Oberflache des Zahns geordnet, vorhanden sind; diese Fibrillen sind anisotrop, und ihre Existenz lässt sich bei Untersuchung der Zahnanlage im polarisierten Licht erweisen. Auch an feinen Schnitten sieht man die Doppeltbrechung. Das Pradentin ist noch isotrop.

Die Bildung des Dentins geschieht in einzelnen Perioden, Lage auf Lage bildet sich und tritt mit dem vorhandenen Dentin in Verbindung. Die Vorgange innerhalb einer jeden Periode sind natürlich die gleichen; zwischen zwei Zeitrdume, in denen Dentin gebildet wird, schiebt sich eine Periode der Ruhe ein, deren Dauer sich aber nicht bestimmen lăsst. Eine solche Ruheperiode charakterisiert sich dadurch, dass die Dentinzellen rein protoplasmatisch erscheinen und keine Spur einer hyalinen Aussenzone erkennen lassen. In einer solchen Ruhezeit sind die Zahnfasern sehr gut zu sehen. Die innere Grenze der Dentinlage enthalt Nischen, in denen die peripheren Enden der Dentinzellen stecken (Fig. $20 \mathrm{~N}$ ); aus diesen Nischen kommt man in die Dentinkanalchen binein, innerhalb deren die Zabnfasern (Fig. 20Zf) gelegen sind. Zwischen den Nischen springt das Dentin mit breiteren und schmaleren Fortsaltzen vor (Fig. 20).

Die jüngste Dentinlage enthalt eine grosse Menge feiner, stark färbbarer Körner; diese verlieren sich plötzlich in einer gewissen Entfernung von der Lage der Dentinzellen.

Die Frage, ob die ganze am fertigen Zahn vorhandene Dentinschicht von einer und derselben Zellenlage gebildet wird, oder ob sich mehrere Zellengenerationen an der Dentinbildung beteiligen, lasst sich durch direkte Üntersuchung nicht entscheiden. Ich bin geneigt, anzunehmen, dass es immer dieselbe 7ellenlage ist, die das Dentin bildet. Man sieht namlich nie Anzeichen dafür, dass Dentinzellen zugrunde gehen; ebensowenig findet man Anzeichen für eine Neubildung von Dentinzellen, etwa mitotische Teilungen einzelner Dentinzellen. Die mit der Verdickung der Dentinlage Schritt haltende Verlangerung der Zahnfaser aber spricht dafür, dass die Dentinzellen in die Lange wachsen, so lange die Dentinbildung dauert. Dann bleibt nur 
übrig, anzunehmen, dass die Umwandlung des peripheren Zellenabschnittes in Prådentin an einer und derselben Dentinzelle vielmal hintereinander sich vollzieht, und dass bei jeder dieser Phasen eine neue Dentinlage gebildet wird, die sich an das vorhandene Dentin anlegt.

Die Vorgănge bei der Dentinbildung, d. h. die Verănderungsstadien an den Dentinzellen sind am leichtesten da zu sehen, wo die Dentinlage am machtigsten wird, also an der Spitze der Zahnpapillen. Hier ist die Dentinbildung sehr rege; demgemăss findet man die Umwandlungsstadien an den Dentinzellen meistens vor und kann sie ihrer Reihefolge nach ordnen.

Dafür liefern die Fig. 15, 16, 17, 18 die Bestătigung. Am freien Rande der Dentinlage ist es schwieriger, die Ver'underungen an den Dentinzellen zu Gesicht zu bekommen; natürlich sind sie auch hier vorhanden, wie Fig. 14 erweist.

Aus den mitgeteilten Beobachtungen folgt, dass das Dentin aus den Dentinzellen hervorgeht. Es ist "umgewandeltes Protoplasma“. Die Umwandlung ergreift die periphere Halfte der Dentinzelle; hier wird der Zellenleib glasartig, homogen, strukturlos. Das in dieser Weise veranderte Protoplasma bleibt mit der protoplasmatisch gebliebenen inneren Zone der Dentinzelle noch einige Zeit in Verbindung, verschmilzt aber mit den gleichartigen Abschnitten der benachbarten Zellen. Dadurch wird eine helle Schicht gebildet, die sich an die Innenflache des fertigen Dentins anlegt.

Diese Schicht trennt sich vom Rest der Dentinzellen ab, wird fester und erbalt eine "provisorische Struktur" Körnchen in ihr auftreten. Dann muss die Schicht als "Prridentin bezeichnet werden. Erst bei der Sonderung des Prädentins wird die Zahnfaser erkennbar; sie stellt den axialen Abschnitt der byalinen Zone einer jeden Dentinzelle vor, der sich nicht in Dentin umwandelt, sondern protoplasmatisch bleibt.

Das Dentin ist anfanglich eine. reine Grundsubstanz; innerbalb dieser erst treten, ganz unabhangig von den Zellen, Bündel leimgebender Fibrillen auf. Es ist verstăndlich, dass diese Grundsubstanz, ein in bestimmter Weise verandertes Zellprotoplasma, weiter lebt, Stoffwechsel besitzt (Schwinden der Körner, Einlagerung von Kalksalzen) und sowohl leimgebende. 
Fibrillen bildet, als auch die sehr widerstandsfähigen Wandungen der Zahnkanalchen produziert.

Die Gleichartigkeit bei der Bildung der Grundsubstanz des Zahmbeins und der Grundsubstanz des Knochengewebes besteht darin, dass beiderlei Grundsubstanzen kein Sekret, sondern umgewandeltes Zellprotoplasma sind. Es bestehen aber bemerkenswerte Unterschiede in der Bildungsweise der genannten Grundsubstanzen. Das hyalin gewordene Protoplasma der Osteoblasten trennt sich völlig von dem protoplasmatisch bleibenden Rest der Zelle $a b$ und wird zur Grundsubstanz des Knochens; bei der Dentinzelle wandelt sich nur die Mantelzone des byalinen Abschnittes in Dentin um, eine axial gelegene Partie bleibt, obgleich sie anfänglich hyalin war, doch protoplasmatisch und erhalt sich als Zahnfaser. Die Grundsubstanz des Knochens sowohl wie die des Dentins bekommt bei ilrrer ersten Bildung Einschlüsse fremdartigen Gewebes.

In das osteogene Gewebe kommen Knorpelreste, oder aber einzelne Bindegewebsfasern hinein; in das Prădentin werden periphere Pulpafasern eingeschlossen. Diese Einschlüsse verschwinden nach kürzerer oder langerer Zeit; sie werden wohl dem neu entstandenen Gewebe völlig assimiliert.

Die Grundsubstanz des Knochens lässt ebenso wie die des Dentins nach ihrer Trennung von den Zellen Bündel leimgebender, bestimmt orientierter Fibrillen entstehen; sie sondert sich ferner gegen die eingeschlossenen Zellen oder Zellfortsătze durch Bildung feiner, sehr widerstandsfäbiger Membranen ab, welche die Wandungen der Knochenhöhlen oder der Zahnkanälchen darstellen. Diese Membranen sind färberisch leicht darstellbar; schon länger war bekannt, dass sie sich in Reagentien erhalten, durch welche die Grundsubstanzen selbst rasch zerstört werden.

Schon Waldeyer hatte angegeben, dass das Protoplasma der Dentinzellen sich in eine homogene, durchsichtige Masse umwandle und dass diese zu Dentin werde (Untersuchungen über die Entwicklung der Zähne. Zeitschrift für rationelle Medizin, III. Reihe, Bd. 24, 1865). „Die Dentinbildung besteht in einer Umwandlung eines Teils des Protoplasma der Elfenbeinzellen 
in leimgebende Su'stanz, mit nachfolgender Verkalkung der letzteren." Waldeyer bildet in Fig. 2 Tafel VI seiner Abhandlung die helle, zwischen den Dentinzellen gelegene Substanz $a b$, die aus dem Protoplasma der Dentinzellen hervorgeht, obne indessen das Bild zu deuten; in Fig. 3 der Tafel mit 1 bezeichnet, ist ein kurzes Stück des homogen gewordenen Protoplasma einer Dentinzelle abgebildet und richtig gedeutet als "die Stelle, wo man einen kontinuierlichen Übergang von Zellprotoplasma der Dentinzelle in die jüngste, noch weiche Dentinlage zieht" (Figurenerklärung, Seite 213).

Diese so wichtigen Hinweise auf sichtbare Verănderungen an den Dentinzellen sind nicht weiter verfolgt worden; noch v. Ebner (Über die Entwicklung der leimgebenden Fibrillen, besonders im Zahnbein. Wiener Sitzungsberichte, math.-naturw. Kl., Bd. 115, Abt. 3, 1906) bringt wenig positive Angaben über die Veränderungen der Dentinzellen bei. Er sagt: "Über die Herkunft des Prädentins kann man sich wohl kaum eine andere Vorstellung machen, als dass dasselbe in erster Linie von den Odontoblasten und deren Fortsätzen gebildet wird. Es tritt an der Basalmembran, zwischen dieser und den oberflächlichen Pulpazellen zuerst zutage, und erscheint wie ein Ausguss zwischen den äussersten Enden der Zellen ... Das Prädentin entsteht auf Kosten der Odontoblasten wahrscheinlich dadurch, dass die Plasmafäden derselben an der Oberflache zu einer chemisch dem Kollagen nahestehenden Substanz, die zunächst nicht fibrillär ist, zerfliessen" (S. 309). Es ist, wie gezeigt wurde, möglich, die Veränderungen an den Odontoblasten zu sehen, und bis zur Ausbildung des Prädentins zu verfolgen.

In einer früheren Mitteilung (Marburger Sitzungsberichte 1907, Juli) war ich zu der Folgerung gekommen, dass die Odontoblasten eine Interzellularsubstanz produzieren, welche die Fahigkeit hat, zu Dentin zu werden. Die Stadien, die der Bildung der Interzellularsubstanz vorausgehen, waren mir damals noch nicht bekannt, und so fehlte der zwingende Beweis dafür, dass diese „Interzellularsubstanz“, d. h. das Prädentin, wirklich von den Dentinzellen gebildet werde.

Beim Knochengewebe, wie beim Zahnbein, bilden die Zellen aus ihrem Protoplasma eine amorphe Grundsubstanz; diese 
ist zuerst da, und lässt durch Differenzierung die leimgebenden Fibrillen entstehen. Diese Strukturen innerbalb der Grundsubstanz entstehen erst, nachdem sich die Grundsubstanz von den Zellen getrennt hat; sie sind also völlig unabhängig von den Zellen, aus welchen die Grundsubstanz gebildet wird. Es liegt nahe, zu fragen, ob dies auch für diejenigen Fasern zutrifft, die in den andern Unterabteilungen der Gruppe der Bindesubstanzen vorkommen. Für die Fasern des fibrillären Bindegewebes hatte bekanntlich Flemming angegeben, dass sie im unveränderten Protoplasma der Bindegewebszellen zuerst vorhanden sind, und dass sie, über den Bereich der Zelle hinausgehend, in die Interzellularsubstanz gelangen. Von einer dem Auftreten der Fasern vorhergehenden Sonderung der Bindegewebszellen in zwei Abschnitte hat Flemming nichts wahrgenommen. Nach seinen Beobachtungen handelte es sich um Bildung von leimgebenden Fasern innerhalb unveränderten Zellprotoplasmas. (Flemming, ZurEntwicklungsgeschichte der Bindegewebsfibrillen. Internationale Beiträge zur wissenschaftl. Medizin, Bd. I, 1891.)

Spatere Beobachter hoben hervor, dass die Anlagen der Fasern nicht innerbalb der Zellen, sondern auf deren Obertlache, in unmittelbarer Nachbarschaft der Zellen, sich befinden; für die Nabelschnur z. B. wird von Golowinski (Anat. Hefte, Bd. 33, 1907) angegeben, dass die Anlagen der Fasern in Form von Reihen von Körnchen auftreten, die auf der Obertläche der Zellen liegen und nach ibrer Ausbildung frei von den Zellen werden. Diese Substanz, welche auf der Zelle liegend die Fahigkeit hat, Fasern zu bilden, ist, nach Laguesse (Sur l'histogénèse de la fibre collagène etc. Archives d'Anatomie microscopique, tome VI, 1003) ein Produkt der betreffenden Zelle; sie ist aufzufassen als eine Oberflächenschicht, entstanden durch Differenzierung des Protoplasma. "Cette Substance n'est autre chose que le cytoplasma même modifié, chimiquement differencié (S. 162). Dieses "modifizierte Cytoplasma" ist dieselbe Substanz, die Hansen (Über die Genese einiger Bindegewebsgrundsubstanzen. Anat. Anzeiger, Bd. 16, 1899) als "Eltoplasma" bezeichnet hat. Das Ektoplasma übernimmt die Bildung leimgebender Fasern.

Nach Hansen, Laguesse, Golowinski entstehen die Bindegewebsfasern in einem chemisch verănderten, auch optisch sich besonders verhaltenden Abschnitte des Zellprotoplasma. Es 
würde die Bildung dieses Abschnittes, des „Elitoplasma“ von Hansen, der Bildung einer hyalinen Zone an den Osteoblasten und an den Dentinzellen, $z u$ vergleichen sein, wenn es festgestellt würde, dass das „Ektoplasma ${ }^{*}$ zur Grundsubstanz des Bindegewebes wird und dass die gesamte Grundsubstanz aus dem Ektoplasma hervorgeht. Diese Frage ist noch nicht geklärt; die mit ihr zusammenbängenden Fragen nach dem Verhalten von Fasern und Grundsubstanz im fibrillären Bindegewebe bedürfeir einer erneuten Bearbeitung. Ist, bevor fasrige Strukturen auftreten, auch im Bindegewebe eine amorphe Grundsubstanz da? Wie verhalt sich diese $z u$ den Zellen, die die erste Anlage des Bindegewebes darstellen? Erst die Beantwortung dieser Fragen wird uns in den Stand setzen, die Bildungsiveise des Bindegewebes einerseits, des Knochens und des Zabnbeins andererseits im einzelnen zu vergleichen.

Marburg, den 10. September 1908.

\section{Erklărung der Abbildungen auf Tafel XXI u. XXII.}

Sämtliche Abbildungen sind bei Zeiss, Apochromat $2 \mathrm{~mm}$, bomogene Immersion, Komp.-Okular 6, gezeichnet.

Fig. 1. Mensch, Embryo des vierten Monats. Tibia. Längsschnitt. Ein Knorpelbalken, mit Osteoblasten belegt. $\mathrm{Kn}=$ Knorpel; $\mathrm{a}, \mathrm{b}, \mathrm{c}=$ die protoplasmatisehen Abschnitte der Osteoblasten; $a_{1}, b_{1}, c_{1}=$ die hyalinen Abschnitte dieser Zellen.

Fig. 2. Mensch, Embryo des vierten Monats. Tibia. Osteoblasten auf einer Lage jungen Knochengewebes, das einen Knorpelrest einschliesst. $\mathrm{Kn}=$ Knorpelrest, Kno $=$ Knochenlage; $\mathrm{Kz}=$ Knochenzelle in einem Hohlraum des jungen Knochens; $a, b=$ protoplasmatische; a1, $\mathrm{b}_{1}=$ byaline Abschnitte zweier Osteoblasten; $c=$ ein Osteoblast dem jungen Knochen aufliegend.

Fig. 3. Mensch, Embryo des vierten Monats. Tibia. Osteoblasten auf einem Knochenbälkchen, das einen Knorpelrest einschliesst. $\mathrm{Kn}=$ Knorpelrest; a, b, $c=0$ Osteoblasten; $a=$ der hyaline Abschnitt des Osteoblasten, a fliesst auf der Oberfläche des Knochenbalkens mit dem hyalinen Abschnitt des Osteoblasten b zusammen.

Fig. 4. Mensch, Embryo des vierten Monats. Tibia. Entstehung von osteugener Substanz auf einem Knorpelbälkchen. $\mathrm{Kn}=\mathrm{Knorpel;} \mathrm{Ks}=$ eine Lage jungen Knochens; $\mathrm{O}_{1} \mathrm{O}_{1}, \mathrm{O}_{2}=$ Osteoblasten; $\mathrm{Gs}_{1}, \mathrm{Gs}_{2}=$ osteogene Substanz, aus den hyalinen Abschnitten der Osteoblasten gebildet. 
Fig. 5. Mensch, Embryo des vierten Monats. Auftreten von Strukturen in den hyalinen Abschnitten der Osteoblasten. $\mathrm{Kn}=\mathrm{Knorpel} ; \mathrm{KS}=$ ihm aufliegende Knochenschicht; $a, b, c=$ Osteoblasten; $b_{1}=$ hyaliner Abschnitt des Osteoblasten $b$, mit Andeutungen einer Faserung.

Fig. 6. Mensch, Embryo des vierten Monats. Deutlichere fasrige Struktur in den hyalinen Abschnitten einiger Osteoblasten. $\mathrm{O}_{1}, \mathrm{O}_{2}, \mathrm{O}_{3}=$ Osteoblasten; in den hyalinen Abschnitten sind Fasern deutlich, die Netze bilden. $\mathrm{Kn}=$ Knorpel.

Fig. 7. Mensch, Embryo des zweiten Monats. Knochenbildung in Membranen. $\mathrm{Kb}=$ ein Knochenbälkchen; $\mathrm{O}_{1}, \mathrm{O}_{2}, \mathrm{O}_{3}=$ Osteoblasten, dem Bälkchen aufliegend; $\mathbf{h}_{1}, \mathbf{h}_{2}, \mathbf{h}_{\mathbf{3}}=$ hyaline Abschnitte dirser Osteoblasten. Der Abschnitt $h_{3}$ füllt ein Grübchen der Knochenoberfläche aus.

Fig. 8. Mensch, Embryo des zweiten Monats. Knochenbildung in Membranen. Das freie Ende des in Fig. 7 dargestellten Knochenbälkchens wächst. durch Anlagerung von osteogener Substanz (Grundsubstanz), die von einer Gruppe von Osteoblasten (Obl) produziert wird. $\mathrm{h}=$ osteogene Substanz, aus den hyalinen Abschnitten der Osteoblasten (Obl) gebildet; $\mathrm{Kb}=$ Knochenbälkchen, durch die Faserzüge ff mit den hyalinen Abschnitten der Osteoblasten (Obl) in Verbindung stehend; $\mathrm{L}=$ Lücke im Knochen, in der zwei Osteoblasten liegen.

Fig. 9. Schwein, Embryo. Anlage des Oberkiefers. Ein Knochenbälkchen (K) mit Osteoblasten belegt. $\quad O_{1} \mathrm{O}_{1}=$ Osteoblasten; $\mathrm{Gs}=$ osteogenes Gewebe (Grundsubstanz), aus den hyalinen Abschnitten der Osteoblasten gebildet. Diese Schicht ist noch mit den Osteoblasten $\left(O_{1}\right)$. im Zusammenhang.

Fig. 10. Mensch, Embryo des zweiten Monats. Belegknochen der Nasenkapsel. In den Lücken des Knochengewebes liegen Osteoblasten $\left(0, O_{1}\right)$, die einen hyalinen Abschnitt ausgebildet haben $\left(h \mathbf{u} . h_{1}\right)$. Der hyaline Abschnitt $\left(h_{1}\right)$ überzieht, wie eine Kappe, den zugehörigen Osteoblasten $(0)$.

Fig. 11. Mensch, Embryo des vierten Monats. Osteogene Substanz (Grundsubstanz), auf einem Knorpelbalken aufliegend, von der Fläche gesehen. $\mathrm{Kn}=$ Knorpelbalken; $\mathrm{O}=$ Osteoblast; Gs = osteogene Substanz, körnige Reste zerfallenen Knorpels ( $\mathrm{Knr} \iota$ ) einschliessend.

Fig. 12. Schwein, Embryo. Partie aus der Umgebang eines in bindegewebiger Grundlage entstandenen Knochenbälkchens. $\mathrm{Kb}=$ Knochenbälkchen, in feine Fäden (b, $c$ ) auslaufend; $a=$ Reihen von Körnern, die zu Fäden zusammentreten; $\cdot d, e=$ Osteoblasten, in deren Umgebung Fadennetze sich ausbilden.

Fig. 13. Mensch, Embryo von $25 \mathrm{~mm}$. Anlage des Oberkiefers. In den Lücken des Knochengewebes $(K)$ liegen Osteoblasten; bei den meisten Zellen ist, ausser dem Kern, nur die hyaline Zone (hZ) sichtbar, nur eine Zelle ist so günstig getroffen, dass man auch die protoplasmatische Zone $(\mathrm{pZ})$ sehen kann.

Fig. 14-20. Dentinbildung. 
Fig. 14. Schwein, Embryo. Anlage eines Schneidezahnes. Längsschnitt. Einige Dentinzellen in der Nähe des freien Randes des Zahnscherbchens sind in einen protoplasmatischen Abschnitt $(\mathrm{pZ})$ und einen hyalinen Abschnitt ( $\mathrm{hZ}$ ) gesondert. $\mathrm{SO}=$ inneres Epithel des Schmelzorgans.

Fig. 14a. Schwein, Embryo. Ein Stück jungen Dentins (D), an seiner Innenfläche Dentinzellen. Jede Dentinzelle besitzt einen protoplasmatischen Abschnitt ( $\mathrm{pZ}$ ) und einen glasartig durchsichtigen, hyalinen $\mathrm{Ab}$ schnitt $(\mathrm{hZ})$.

Fig. 15. Mensch, Embryo von $140 \mathrm{~mm}$. Sonderung der Dentinzellen in zwei Abschnitte. $\mathrm{pZ}=$ protoplasmatischer Abschnitt mit endständigem Kern ; $h \mathrm{Z}=$ hyaliner Abschnitt, dem Dentin (D) anliegend.

Fig. 16. Mensch, Embryo von $132 \mathrm{~mm}$. Einige Dentinzellen an der Spitze der Zahnpapille. $\mathrm{pZ}=$ protoplasmatische $\mathrm{Z}$ one; $\mathrm{h} Z=$ hyaline Zone; $\mathrm{D}=$ Dentinlage, deren Bildung gerade beginnt.

Fig. 17. Mensch, Embryo von $140 \mathrm{~mm}$. Schneidezahn, Längsschnitt. Dentinzellen an der Innenfläche der Dentinlage (D). $\mathrm{h} Z=$ hyaline Aussenzone der Dentinzellen.

Fig. 18. Mensch, Embryo von $140 \mathrm{~mm}$. Schneidezahn. Die hyaline Aussenzone hat sich von dem Protoplasma der Dentinzellen (DZ) getrennt und ist zu einer einheitlichen Schicht, dem Prädentin (PD), geworden. Die Zahnfasern (Zf) sind deutlich.

Fig. 19. Mensch, Embryo aus dem fünften Monat. Längsschnitt der Anlage eines Schneidezahns. Das Prädentin (PD) wird fest, erscheint körnig; enthält Hohlräume $(R)$, in denen die Dentinzellen stecken. $\mathrm{HZ}=$ die noch homogenen Abschnitte des Prädentins; $\mathrm{D}=$ fertiges Dentin; $\mathrm{DZ}=$ Dentinzellen.

Fig. 20. Mensch, Embryo aus dem fünften Monat. Junges und älteres Dentin. $\mathrm{D}=$ älteres; $\mathrm{DI}=$ jüngeres Dentin; $\mathrm{Zf}=$ Zahnfasern; $\mathrm{DZ}=$ Dentinzellen; $\mathrm{Cg}=$ Kapillargefäss; $\mathrm{N}=$ Nische für das periphere Ende einer Dentinzelle. Längsschnitt der Anlage eines Schneidezahns. 


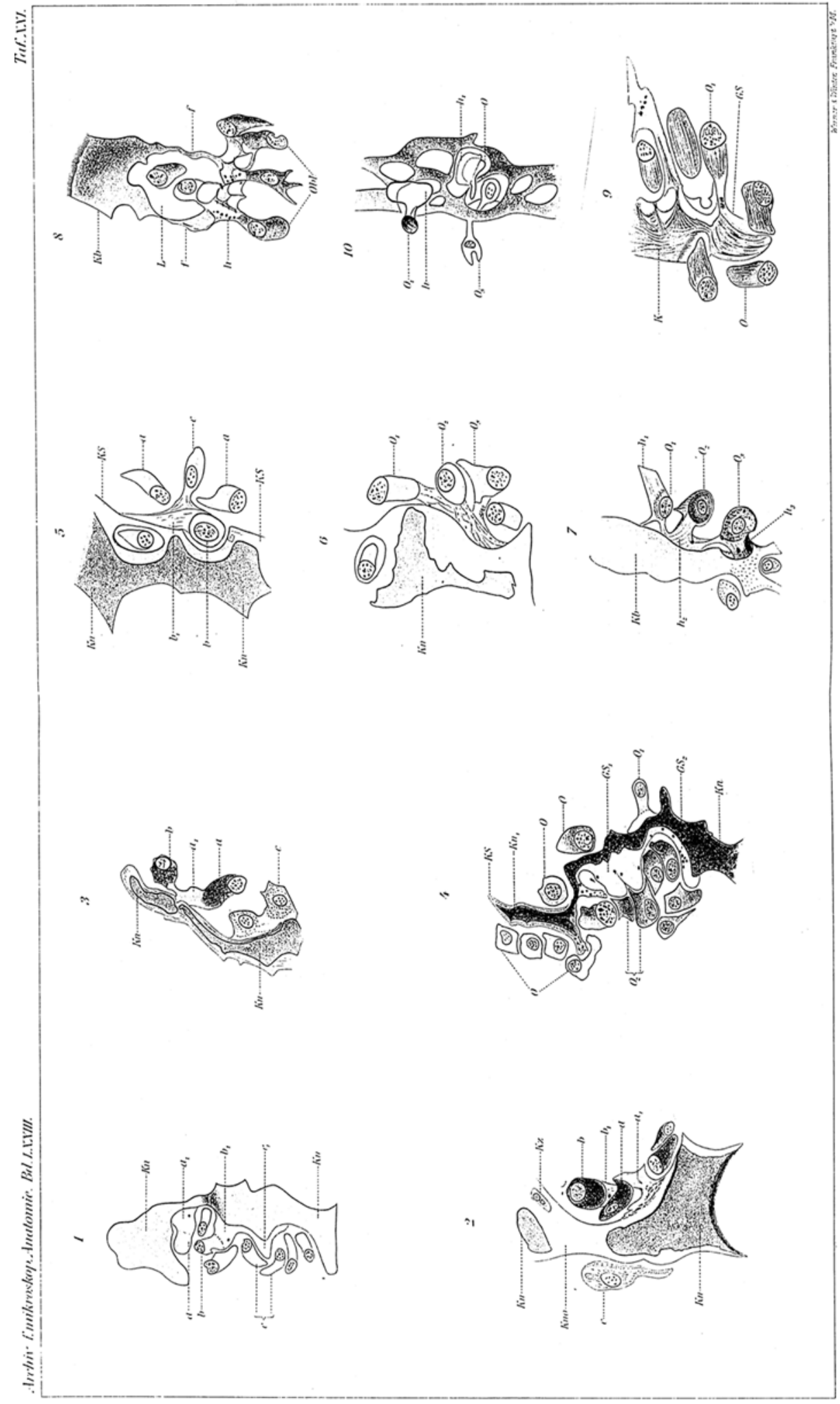




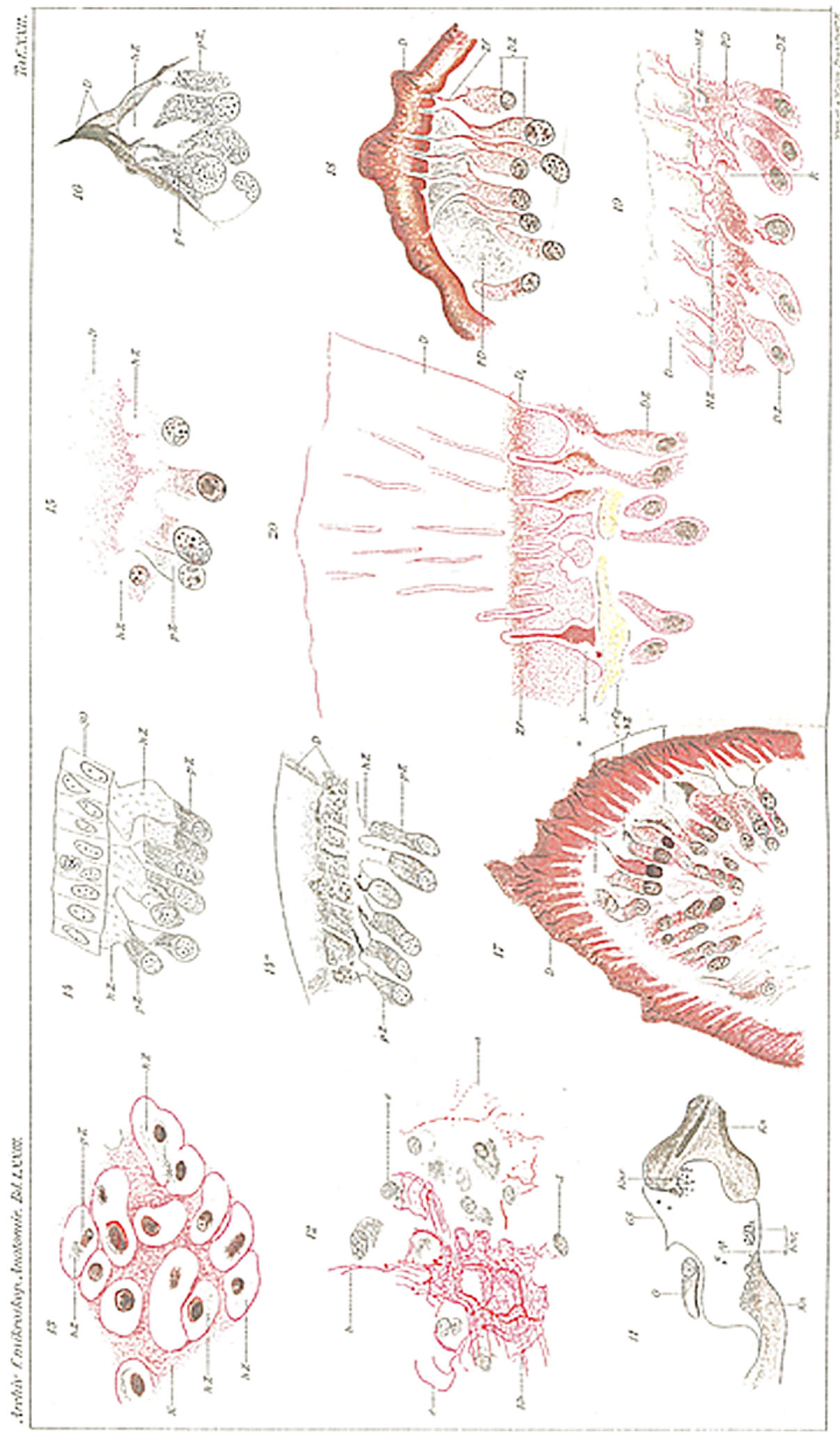

